\title{
Ciclos Hamiltonianos em Grafos
}

\section{Hamiltonian Cycles in Graphs}

\author{
Marcelo de Souza Santos \\ marcelosantos@ufrgs.br
}

Universidade Federal do Rio Grande do Sul, RS, Brasil

\begin{abstract}
Resumo
Neste trabalho tratamos de um problema clássico bem conhecido em Teoria dos Grafos: o problema da existência de um ciclo hamiltoniano. Um grafo é dito hamiltoniano se possui um ciclo hamiltoniano, ou seja, apresenta um ciclo que percorre todos os vértices do grafo. Estudamos problemas clássicos associados a este problema em termos do número de arestas, do grau mínimo e da sequência de graus dos vértices de um grafo. Além disso, estudamos resultados espectrais para o problema de hamiltonicidade referentes às matrizes de adjacências e laplaciana. A principal contribuição deste trabalho é a apresentação detalhada de condições suficientes e condições necessárias que garantem um ciclo hamiltoniano em um grafo já existentes na bibliografia.
\end{abstract}

Palavras-chave: Ciclos hamiltonianos, Teoria Espectral de Grafos, Grafos.

\section{Abstract}

In this work we study a classic problem well know in Graph Theory: the existence of a Hamiltonian cycle. A graph is called hamiltonian if it has a hamiltonian cycle, it means, if presents a cycle that walks every vertex of the graph (without repetition). We study classic problems related with this problem in terms of the number of edges, the minimal degree and the vertex degree sequence of a graph. Furthermore, we study spectral results for the hamiltonian problem related to the adjacency and laplacian matrix. The main contribution of this work is present detailed necessary and sufficient conditions that ensures a hamiltonian cycle in a graph that are already in bibliography.

Keywords: Hamiltonian cycles; Spectral Graph Theory; Graphs. 


\section{Introducão}

A Teoria dos Grafos é um ramo da Matemática que estuda as relações entre os objetos de um determinado conjunto. Para tal estudo, são utilizados estruturas que chamamos de grafos. Seja $G=(V, E)$ um grafo, onde $V$ é um conjunto finito cujos elementos são denominados vértices e $E$ é um conjunto de subconjuntos de dois elementos de $V$ denominados arestas. Podemos representar muitas situações reais através de grafos, por exemplo, a Internet, onde os vértices são os sites e as arestas são links entre os sites. O Facebook também pode ser facilmente modelado por um grafo, cujos vértices são perfis e cujas arestas são relações de amizade.

Na Teoria dos Grafos, podemos destacar problemas clássicos como o Problema das Pontes de Königsberg e o Problema das Quatro Cores. O primeiro problema trata de realizar um passeio pela cidade de Königsberg passando por suas sete pontes, sem repetir nenhuma, e retornando ao ponto de partida. Em 1741, Euler (1741) resolveu esse problema utilizando o seguinte raciocínio: ele considerou as pontes como as arestas e as ilhas ou margens como vértices, criando assim um multigrafo, apresentado na figura abaixo.

Euler mostrou que um grafo conexo G possui um circuito que passa, exatamente uma vez, por todas as arestas se, e somente se, todos os seus vértices são incidentes a um número par de arestas. Logo o problema das pontes de Königsberg não tem solução, pois todos os vértices do grafo da Figura 1 têm um número ímpar de arestas incidentes. Posteriormente, tais grafos ficaram conhecidos como grafos eulerianos.

O Problema das Quatro Cores trata da afirmação de que quatro cores bastariam para colorir um mapa qualquer de tal maneira que países vizinhos não tenham a mesma cor, como conjecturado por Guthrie. Tal problema surgiu por volta de 1852 e, durante mais de cem anos, muitos métodos foram desenvolvidos para resolvê-lo, mas foi apenas em 1976 que Appel e Haken (1976) apresentaram uma demonstração (com auxílio computacional importante) do Teorema das Quatro Cores. O estudo desse problema teve um papel muito importante no desenvolvimento da Teoria dos Grafos.

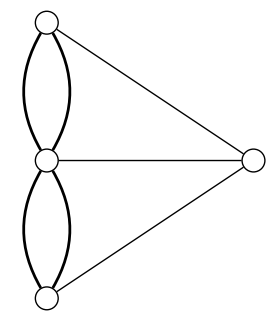

Figura 1: Grafo modelo das sete pontes de Königsberg.

O objetivo deste trabalho é investigar as diversas ferramentas combinatórias para estudar um outro problema clássico bem conhecido em Teoria dos Grafos, o problema da existência de um ciclo hamiltoniano. Um caminho e um ciclo que percorram todos os vértices de um grafo (não repetindo nenhum vértice) são chamados, respectivamente, de caminho hamiltoniano e ciclo hamiltoniano de um grafo. Um grafo é hamiltoniano se contém um ciclo hamiltoniano.

O dodecaedro é hamiltoniano, pois possui um ciclo hamiltoniano e o grafo de Herschel não é hamiltoniano mas possui um caminho hamiltoniano. A Figura 2 apresenta um ciclo hamiltoniano no dodecaedro e um caminho hamiltoniano no grafo de Herschel.
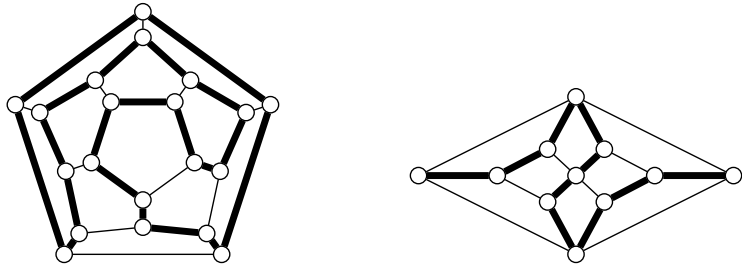

Figura 2: Dodecaedro (hamiltoniano) e Grafo de Herschel (não-hamiltoniano).

Apesar de ser um problema extremamente simples de enunciar, decidir se um grafo é hamiltoniano é $N P$ completo (tempo polinomial não determinístico) Karp (1972), o que sugere que seja extremamente difícil encontrar 
uma caracterização simples de grafos hamiltonianos. Conseguir essa caracterização significa encontrar condições necessárias e suficientes para que um grafo seja hamiltoniano que permitam decidir eficientemente se um grafo é hamiltoniano, encontrando um ciclo hamiltoniano se a resposta for positiva ou uma evidência simples de que isso não é possível se a resposta for negativa.

$\mathrm{Na}$ ausência de uma caracterização simples de grafos hamiltonianos, muito da pesquisa nessa área consiste em estudar condições necessárias ou condições suficientes para que um grafo seja hamiltoniano. Além de fazer um apanhado de condições clássicas, este trabalho estuda condições espectrais relacionadas a este problema.

O ramo da Teoria dos Grafos que se dedica ao estudo de propriedades de um grafo por meio de suas representações matriciais e de seus respectivos espectros é denominado Teoria Espectral de Grafos. Dado um grafo, conseguimos associá-lo a matrizes, e o espectro dessas matrizes está diretamente ligado a propriedades estruturais do grafo. Dentre as representações matriciais de grafos que serão utilizadas neste trabalho, estão a Matriz de Adjacências e a Matriz Laplaciana.

A Teoria Espectral de Grafos teve sua origem motivada pela Química Quântica, por Hückel (1931), que estabeleceu as ideias iniciais da teoria ao representar uma molécula de hidrocarboneto por um grafo e verificar a relação entre os autovalores do grafo e a energia de elétrons associados à molécula. Porém, somente em 1971, esse estudo foi trazido para o meio matemático e computacional por Cvetković (1971).

\section{Conceitos Preliminares}

Nesta seção, apresentamos conceitos básicos de Teoria dos Grafos, Álgebra Linear e Teoria Espectral de Grafos que serão utilizados ao longo deste trabalho. Tais definições, retiradas de Abreu et al. (2014), Bondy e Murty (2008), Meyer (2000), Brouwer e Haemers (2012), serão importantes para a compreensão dos resultados apresentados.

\subsection{Grafos}

Muitas situações do mundo real podem ser descritas através de diagramas consistindo de um conjunto de pontos e de um conjunto de linhas que ligam certos pares destes pontos. Por exemplo, os pontos poderiam representar pessoas e as linhas pares de amigos, ou os pontos podem ser centros de comunicação com linhas que representam a ligação entre os centros. O interessante nesses diagramas é saber se os pontos são ligados ou não por linhas. Situações matemáticas deste tipo deram origem ao conceito de grafo.

Um grafo é uma estrutura $G=(V, E)$, constituída por um conjunto finito e não vazio $V$ cujos elementos são denominados vértices, e por um conjunto $E$ de subconjuntos de dois elementos de $V$, denominados arestas. Indicamos por $|V|$ e $|E|$, respectivamente, o número de vértices e o número de arestas de G.

Os vértices de um grafo são representados graficamente por pontos e as arestas por ligações entre esses pontos, como mostra a Figura 3. Neste trabalho, consideramos apenas grafos simples, ou seja, sem arestas paralelas (duas ou mais ligações entre o mesmo par de vértices) e sem laços (arestas que ligam um vértice com ele mesmo). Dado um grafo $G=(V, E)$ e $u, v \in \mathrm{V}$, dizemos que a aresta $e=\{u, v\} \in E$ é incidente em $u$ e $v$. Vértices pertencentes a uma mesma aresta são ditos adjacentes ou vizinhos.

Definição 2.1. Seja $G=(V, E)$ um grafo. O grau de um vértice v, denotado por $d(v)$, é o número de arestas que incidem em v. O grau mínimo de $G$, denotado por $\delta(G)$, é o número $\delta=\min \{d(v): v \in V\}$ e o grau máximo de $G$, denotado por $\Delta(G)$, é o número $\Delta=\max \{d(v): v \in V\}$.

Exemplo 2.1. No grafo representado na Figura 3, temos $V=\left\{v_{1}, v_{2}, v_{3}, v_{4}\right\}$ e $E=\left\{e_{1}, e_{2}, e_{3}, e_{4}, e_{5}\right\}$, e portanto $|V|=4$ e $|E|=5$. Os graus dos vértices são: $d\left(v_{1}\right)=d\left(v_{3}\right)=2$ e $d\left(v_{2}\right)=d\left(v_{4}\right)=3$. O grau mínimo de $G$ é $\delta(G)=2$ e o grau máximo é $\Delta(G)=3$.

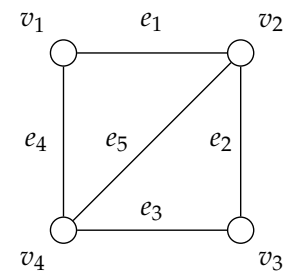

Figura 3: Grafo do Exemplo 2.1.2. 
Definição 2.2. Um grafo é dito regular se todos os seus vértices têm o mesmo grau. Um grafo regular com vértices de grau ké chamado de grafo k-regular.

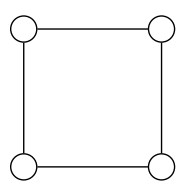

Figura 4: Um grafo 2-regular.

Definição 2.3. Dado um grafo $G=(V, E)$, dizemos que $H=(W, F)$ é um subgrafo de $G$ se $H$ é um grafo, $W \subseteq V$ e $F \subseteq E$. No caso em que $F=\{\{u, v\} \in E: u, v \in W\}$, dizemos que $H$ é o subgrafo induzido de $W$, denotado $H[W]$.

Na Figura 5, apresentamos em (A) um grafo G, em (B) um subgrafo de G e em (C) um subgrafo induzido de G.

Definição 2.4. Um grafo no qual quaisquer dois vértices distintos são adjacentes é dito grafo completo. Denotamos por $K_{n} o$ grafo completo com $n$ vértices.

A Figura 6 representa o grafo completo $K_{5}$.

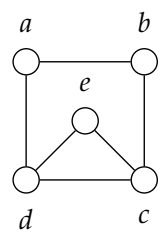

(A)

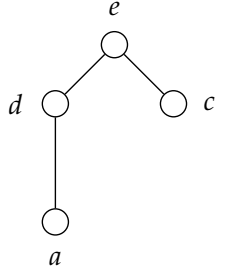

(B)

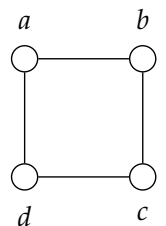

(C)

Figura 5: Grafo, subgrafo e subgrafo induzido.

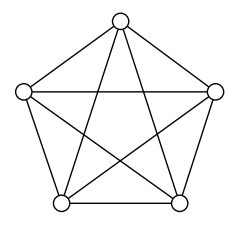

Figura 6: Grafo Completo $K_{5}$.

Definição 2.5. Um passeio entre dois vértices $u, v \in V$ é uma sequência finita $u=v_{1}, v_{2}, v_{3}, \ldots, v_{n}=v$ de vértices de um grafo $G=(V, E)$ tal que $e_{i}=\left\{v_{i}, v_{i+1}\right\} \in E$ para $1 \leq i \leq n-1$. Quando, nessa sequência, temos $v_{1}=v_{n}$, chamamos de um passeio fechado.

Definição 2.6. Um caminho é um passeio sem repetição de vértices, e um ciclo é a união de um caminho com pelo menos duas aresta entre as suas pontas.

O comprimento de um caminho ou de um ciclo é o número de arestas que ocorrem em cada um. Os únicos caminho e ciclo com $n$ vértices, a menos de isomorfismo, são denotados, respectivamente, por $P_{n}$ e $C_{n}$. 
Definição 2.7. Um grafo é conexo se existe um caminho ligando cada par de vértices. Caso contrário, o grafo é denominado desconexo.

Se $G$ é um grafo desconexo, dizemos que $G^{\prime} \subset G$ é uma componente conexa de $G$ quando $G^{\prime}$ é um subgrafo conexo maximal de $G$, isto é, $G^{\prime}$ é conexo e não existe um grafo conexo $H \subset G$ tal que $G^{\prime} \subset H$ e $G^{\prime} \neq H$. Exemplos de grafos conexos e desconexos estão na Figura 7.
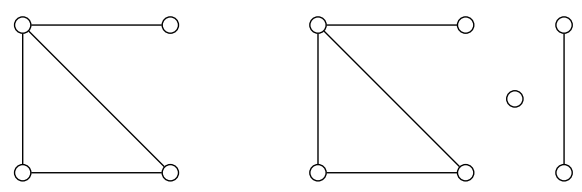

Figura 7: Grafo conexo e grafo desconexo com três componentes conexas.

Definição 2.8. Dado um grafo $G=(V, E)$, o grafo complementar de $G$, denotado por $\bar{G}=(\bar{V}, \bar{E})$, é o grafo com o mesmo conjunto de vértices de $G$ e tal que $e=\{u, v\} \in \bar{E}$ se, e somente se, $e=\{u, v\} \notin E$.

Definição 2.9. Um grafo é dito bipartido se seu conjunto de vértices pode ser particionado em dois subconjuntos X e $Y$ tais que todas as arestas têm uma extremidade em X e uma extremidade em $Y$. Um grafo bipartido é dito completo se todos os vértices do conjunto $X$ forem adjacentes a todos os vértices do conjunto $Y$. Nesse caso, se $|X|=r$ e $|Y|=s$, escrevemos $G=K_{r, s}$. Um grafo do tipo $G=K_{1, n}$ é chamado estrela.
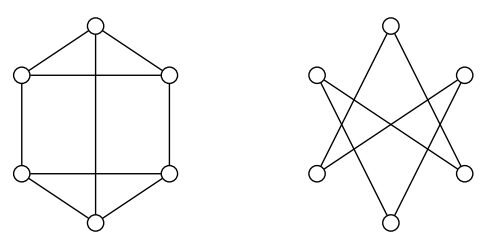

Figura 8: Grafo e seu complementar.
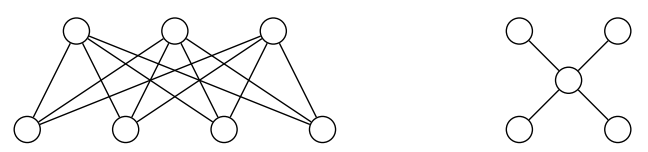

Figura 9: $K_{3,4}$ e $K_{1,4}$.

\section{2 Álgebra Linear}

Muitos conceitos de Álgebra Linear serão importantes para a compreensão das condições que garantem a hamiltonicidade em grafos, principalmente quando começarmos a associar diferentes matrizes a um grafo e a estudar as propriedades dos grafos através dessas matrizes. Nesta seção, apresentaremos algumas definições e resultados que foram relevantes no trabalho.

Dada uma matriz simétrica $M_{n \times n}$, os autovalores são dados pelo conjunto $\left\{\lambda_{1}^{M}, \lambda_{2}^{M}, \ldots, \lambda_{n}^{M}\right\}$ e, por serem reais, podemos ordenar tais autovalores de tal forma que $\lambda_{1}^{M} \geq \lambda_{2}^{M} \geq \cdots \geq \lambda_{n}^{M}$.

Definição 2.10. Dada uma matriz $M$, chamamos de espectro de Mo multiconjunto formado pelos autovalores da matriz M e suas respectivas multiplicidades. Denotamos o espectro da matriz $M$ como spect $(M)$.

Definição 2.11. Uma matriz P é dita matriz de permutação se gera uma permutação dos elementos de um vetor ou entre linhas ou colunas de uma matriz. 
Definição 2.12. Uma matriz simétrica $A_{n \times n}$ é redutível quando existe uma matriz de permutação $P_{n \times n}$ e um inteiro $r$ tais que $1 \leq r \leq(n-1) e$

$$
P^{T} A P=\left[\begin{array}{cc}
X & Y \\
0 & Z
\end{array}\right]
$$

onde $X$ e $Z$ são matrizes quadradas de ordem $r \times r e(n-r) \times(n-r)$, respectivamente, Y é uma matriz de ordem $r \times(n-r)$ e 0 é a matriz nula de ordem $(n-r) \times r$. Caso contrário, A é dita uma matriz irredutível.

Teorema 2.1 (Perron-Frobenius). (Abreu et al., 2014, Teorema 7.4) Seja a matriz $A_{n \times n}$ não negativa, irredutível e com autovalores $\lambda_{1} \geq \lambda_{2} \geq \cdots \geq \lambda_{n}$. Então

(i) $\lambda_{1}>0$ e existe um autovetor não-nulo $x$ tal que $A x=\lambda_{1} x$;

(ii) $\lambda_{1}>\lambda_{2}$;

(iii) $\left|\lambda_{i}\right| \leq \lambda_{1}$, para todo $i \in 1,2, \ldots, n$;

(iv) o único vetor definido por $A p=\lambda_{1} p, p>0 e\|p\|_{1}=1$ é chamado vetor de Perron. Não existe um autovetor não-negativo para A exceto para múltiplos positivos de $p$, independentemente do autovalor.

Definição 2.13. Seja um vetor $x \in \mathbb{R}^{n}$. Para $p \geq 1$ a p-norma de $x$ é definida como

$$
\|x\|_{p}=\left(\sum_{i=1}^{n}\left|x_{i}\right|^{p}\right)^{1 / p} .
$$

Os resultados abaixo são conhecidas como Princípio de Rayleigh. Elas caracterizam os autovalores de uma matriz $A$ como valores máximos de uma forma quadrática restrita à esfera unitária de $\mathbb{R}^{n}$ com centro na origem.

Proposição 2.1. (Abreu et al., 2014, Proposição 7.2) Seja $A_{n \times n}$ simétrica. Então:

$$
\lambda_{1}^{A}=\max _{\|x\|=1}\langle A x, x\rangle
$$

Corolário 2.1. Seja $A_{n \times n}$ simétrica. Então

$$
\lambda_{1}^{A}=\max _{x \neq 0} \frac{\langle A x, x\rangle}{\langle x, x\rangle},
$$

e o valor máximo é atingido somente em autovetores unitários associados ao autovalor $\lambda_{1}^{A}$.

\subsection{Teoria Espectral de Grafos}

Dado um grafo $G$, podemos associar a ele diferentes matrizes. A Teoria Espectral de Grafos estuda as propriedades do grafo por meio dessas representações matriciais e de seus respectivos espectros. Nesta seção, apresentamos matrizes associadas a grafos e resultados obtidos na literatura acerca do espectro dessas matrizes, que serão utilizados em nosso estudo sobre o problema de hamiltonicidade em grafo.

Definição 2.14. Seja $G=(V, E)$ um grafo com $n$ vértices $\left\{v_{1}, \ldots, v_{n}\right\}$. A matriz de adjacências $A(G)$ de $G$ é a matriz quadrada de ordem $n$ cujas entradas são

$$
a_{i j}= \begin{cases}1, & \text { se } v_{i}, v_{j} \in V \text { são adjacentes, } \\ 0, & \text { caso contrário. }\end{cases}
$$

A matriz de adjacências $A(G)$ é uma matriz real formada por zero e uns. Essa matriz é simétrica, pois a qualidade de vértices serem adjacentes é simétrica. Sendo assim, os autovalores da matriz $A(G)$ são reais. Como estamos trabalhando apenas com grafos simples, o traço de $A(G)$ é zero, então a soma dos seus autovalores é zero.

Observe que, pela definição, a soma dos elementos de cada linha ou coluna da matriz de adjacências de um grafo é igual ao grau do vértice correspondente. Na Figura 10, exibimos um grafo $G$ e sua matriz de adjacências $A(G)$. 


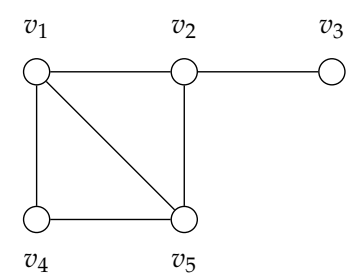

\begin{tabular}{|c|c|c|c|c|c|}
\hline & $v_{1}$ & $v_{2}$ & $v_{3}$ & $v_{4}$ & $v_{5}$ \\
\hline$v_{1}$ & {$[0$} & 1 & 0 & 1 & \\
\hline$v_{2}$ & 1 & 0 & 1 & 0 & 1 \\
\hline$v_{3}$ & 0 & 1 & 0 & 0 & \\
\hline$v_{4}$ & 1 & 0 & 0 & 0 & \\
\hline$v_{5}$ & $L_{1}$ & 1 & 0 & 1 & \\
\hline
\end{tabular}

Figura 10: Grafo $G$ e a respectiva matriz de adjacências $A(G)$.

As raízes do polinômio característico da matriz de adjacências são os autovalores do grafo $G$. Denotamos por $\operatorname{spect}(G)$ o espectro do grafo $G$. O maior autovalor da matriz de adjacências $A(G)$ de um grafo $G$ é denominado índice de $G$ é denotado por $\lambda_{1}^{A}$.

O grafo $G$, representado na Figura 10, tem como polinômio característico $P_{G}(\lambda)=\lambda^{5}-6 \lambda^{3}-4 \lambda^{2}+3 \lambda+2$. As suas raízes são todas simples e os autovalores são aproximados por $-1.7751,-0.5892,-1,0.7237,2.6412$. $\mathrm{O}$ índice de $G$ é $\lambda_{1}^{A}=2.6412$.

Definição 2.15. O raio espectral é o número real $\mu(G)=\max \left\{\left|\lambda_{i}\right|: i=1,2, \ldots, n\right\}$, onde $\lambda_{1}, \ldots, \lambda_{n}$ são autovalores. Isso éo raio do menor intervalo de centro na origem que contém todos os autovalores.

Se o grafo $G$ for conexo, o item (i) do Teorema de Perron-Frobenius garante que o raio espectral é o índice do grafo, pois grafos conexos possuem matrizes de adjacências irredutíveis. O teorema garante também que $\mu(G)$ é positivo e tem multiplicidade 1.

Definição 2.16. Seja $D$ a matriz diagonal dos graus dos vértices $\left\{v_{1}, \ldots, v_{n}\right\}$ de um grafo $G$ (ou seja, a matriz $D$ tal que $D_{i i}=d\left(v_{i}\right)$ ) e seja $A(G)$ a matriz de adjacências de $G$. A matriz $L=D-A$ é a matriz laplaciana do grafo $G$.

Na Figura 11, temos um exemplo das matrizes de adjacências, diagonal e laplaciana de um grafo G.

$$
A(G)=\left[\begin{array}{lllll}
0 & 1 & 1 & 1 & 0 \\
1 & 0 & 0 & 1 & 0 \\
1 & 0 & 0 & 0 & 0 \\
1 & 1 & 0 & 0 & 1 \\
0 & 0 & 0 & 1 & 0
\end{array}\right]
$$

Figura 11: Grafo $G$ e as matrizes $A(G), D(G)$ e $L(G)$. 
A seguir, apresentamos um resultado que relaciona os autovalores da matriz de adjacências com os autovalores da matriz laplaciana para um grafo $d$-regular.

Proposição 2.2. Seja $G$ um grafo d-regular com $n$ vértices, e seja $x \in \mathbb{R}^{n}$ um vetor não nulo. Temos que $x$ é um autovetor da matriz $A(G)$, associado ao autovalor $\lambda^{A}$, se, e somente se, $x$ é um autovetor da matriz $L$, associado ao autovalor $d-\lambda^{A}$.

Demonstração. Supomos que $x$ é um autovetor da matriz de adjacências associado ao autovalor $\lambda^{A}$. Assim, como $L=D-A$, temos que $A=D-L$. Dessa forma, temos:

$$
\begin{gathered}
A x=\lambda^{A} x \Rightarrow(D-L) x=\lambda^{A} x \Rightarrow L x=D x-\lambda^{A} x \\
\Rightarrow L x=\left(d-\lambda^{A}\right) x .
\end{gathered}
$$

Portanto, $x$ é autovetor de $L$ associado ao autovalor $d-\lambda^{A}$. A recíproca é análoga.

Posteriormente, iremos utilizar um resultado de Cvetković et al. (2010) sobre o espectro de um grafo bipartido completo, particularmente de um grafo estrela. Temos que, para um grafo bipartido completo $K_{n_{1}, n_{2}}$, seu polinômio característico é dado por:

$$
P_{K_{n_{1}, n_{2}}}(x)=\left(x^{2}-n_{1} n_{2}\right) x^{n_{1}+n_{2}-2} \text {. }
$$

Para o caso particular onde $n_{1}=n$ e $n_{2}=1$, obtemos um grafo estrela de $n+1$ vértices cujo polinômio característico é dado por:

$$
P_{K_{1, n}}(x)=\left(x^{2}-n\right) x^{n-1} .
$$

Portanto, temos que $\mu\left(K_{1 . n-1}\right)=\sqrt{n-1}$ associado ao autovetor $x=(\sqrt{n-1}, 1,1, \ldots, 1)$.

Também utilizaremos o resultado abaixo para o raio espectral de um grafo $G$ obtido de $K_{1, n-1}$ com adição de algumas arestas:

$$
\mu(G)>\mu\left(K_{1, n-1}\right)=\sqrt{n-1} .
$$

O fato que $\mu(G) \geq \mu\left(K_{1, n-1}\right)$ é imediato, pois $K_{1, n-1}$ é um subgrafo de $G$. Para provarmos a desigualdade estrita em (1), utilizaremos o Princípio de Rayleigh, que afirma que

$$
\lambda_{1}^{A}=\max _{x \neq 0} \frac{\langle A x, x\rangle}{\langle x, x\rangle}=\max _{x \neq 0} \frac{x^{T} A x}{x^{T} x}
$$

e o valor máximo é atingido quando $x$ é autovetor unitário associado $\lambda_{1}^{A}$.

Desenvolvendo o produto interno $\langle A x, x\rangle=x^{T} A x$, onde $x=\left(x_{i}\right)$ e $A=\left(a_{i j}\right)$, temos que:

$$
\begin{aligned}
x^{T} A x & =x_{1} \cdot \sum_{r=1}^{n} x_{r} a_{1 r}+x_{2} \cdot \sum_{r=1}^{n} x_{r} a_{2 r}+\cdots+x_{n} \cdot \sum_{r=1}^{n} x_{r} a_{n r} \\
& =\sum_{i=1}^{n} \sum_{j=1}^{n} a_{i j} x_{i} x_{j} .
\end{aligned}
$$

Como o nosso autovetor $x$ associado ao maior autovalor do grafo estrela tem entradas todas positivas, ao criarmos mais entradas $a_{i j} \neq 0$, o valor $x^{T} A x$ será maior do que o valor que tinhamos anteriormente. Portanto

$$
\mu(G) \geq\langle A(G) x, x\rangle>\left\langle A\left(K_{1, n-1}\right) x, x\right\rangle=\sqrt{n-1} .
$$

\section{Condições clássicas de Hamiltonicidade}

Um problema clássico em Teoria dos Grafos é o da existência de um ciclo hamiltoniano. Dado um grafo $G=(V, E)$, o problema é determinar se $G$ possui um ciclo hamiltoniano, isto é, um ciclo que, partindo de um vértice qualquer, percorre todos os demais vértices, sem repetição, e retorna ao vértice inicial.

É difícil decidir se um grafo $G$ é hamiltoniano. Devido a sua complexidade, esse é um dos problemas que constam na primeira lista de problemas NP-completos Karp (1972). A NP-completude do problema sugere que seja extremamente difícil encontrar condições necessárias e suficientes para que um grafo seja hamiltoniano. A busca de tal caracterização é um dos maiores problemas em aberto da Teoria dos Grafos. 
Muito da pesquisa relacionada a esse problema consiste em encontrar condições suficientes ou necessárias para que um grafo seja hamiltoniano. São conhecidas condições justas que serão apresentadas nessa seção que garantem a hamiltonicidade de um grafo em termos do número de arestas, do grau mínimo e da sequência de graus dos vértices. As demonstrações nessa seção foram adaptadas de Bondy e Murty (2008); West (1996).

\subsection{Condições Suficientes}

Lema 3.1. Seja $G$ um grafo de $n$ vértices com grau mínimo $\delta(G) \geq \frac{(n-1)}{2}$. Então G é conexo.

Demonstração. Se $G$ tem apenas um vértice, então ele é trivialmente conexo. Se $G$ tem dois ou mais vértices e grau mínimo $\delta(G) \geq \frac{(n-1)}{2}$, suponhamos, para obter uma contradição, que $G$ será desconexo. Então segue que $G$ possui $m \geq 2$ componentes conexas.

Sejam $\underbrace{G_{1}, G_{2}, \ldots, G_{m}}_{m \text { componentes }}$, com $n_{1}, n_{2}, \ldots, n_{m}$ vértices, respectivamente. Para cada $i, 1 \leq i \leq m$, tomamos um vértice $x_{i} \in V\left(G_{i}\right)$. Logo $\delta\left(x_{i}\right) \geq \frac{(n-1)}{2}$, por hipótese. Então, segue que, para todo $i, n_{i} \geq \frac{(n-1)}{2}+1$, ou seja, $n_{i} \geq \frac{(n+1)}{2}$.

Assim, $n_{1}+n_{2}+\ldots+n_{m} \geq m \frac{(n+1)}{2}$. Portanto, $n=n_{1}+n_{2}+\ldots+n_{m} \geq m \frac{(n+1)}{2} \geq 2 \frac{(n+1)}{2}=n+1$, chegamos à contradição desejada. Logo $G$ é conexo.

Teorema 3.1 (Dirac (1952)). Se Gé um grafo com $n \geq 3$ vértices e grau mínimo $\delta(G) \geq \frac{n}{2}$, então $G$ é hamiltoniano.

Demonstração. Vamos fazer uma demonstração por absurdo. Suponhamos que $G$ não seja hamiltoniano. Tomamos $P$ um caminho $v_{1} v_{2} \ldots v_{p}$ em $G$ com comprimento máximo.

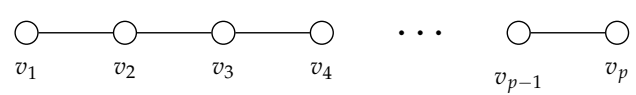

Portanto, todo vértice adjacente a $v_{1}$ e todo vértice adjacente a $v_{p}$ pertence a $P$. Dessa forma, $d\left(v_{1}\right)$ e $d\left(v_{p}\right)$ são, no máximo, $p-1$. Afirmamos que deve existir algum $i, 1 \leq i \leq p-1$ tal que temos $v_{1}$ adjacente a $v_{i+1}$ e $v_{i}$ adjacente a $v_{p}$.

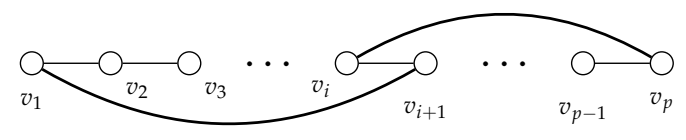

Suponhamos que este não seja o caso, ou seja, se $v_{i}$ é adjacente a $v_{p}$ então $v_{i+1}$ não é adjacente a $v_{1}$. Portanto,

$$
d\left(v_{p}\right) \leq p-1-d\left(v_{1}\right) \Longrightarrow d\left(v_{1}\right)+d\left(v_{p}\right) \leq p-1 .
$$

Assim, $d\left(v_{p}\right) \leq p-1-\frac{n}{2}<n-\frac{n}{2}=\frac{n}{2}$. Isso é absurdo, pois, por hipótese, $\delta(G) \geq \frac{n}{2}$.

Agora tomamos o ciclo $C=v_{1} v_{2} \ldots v_{i} v_{p} v_{p-1} \ldots v_{i+1} v_{1}$ com os mesmos vértices de $P$, porém utilizando as arestas $v_{i} v_{p}, v_{i+1} v_{1}$. Como $G$ é não hamiltoniano, existe no mínimo um vértice $w$ de $G$ que não está em $P$. Mas como $\delta(G) \geq \frac{n}{2} \geq \frac{(n-1)}{2}$, pelo Lema 3.1, G é conexo e portanto podemos escolher um $w$ com essa propriedade que seja adjacente a algum vértice $v_{i} \in P$.

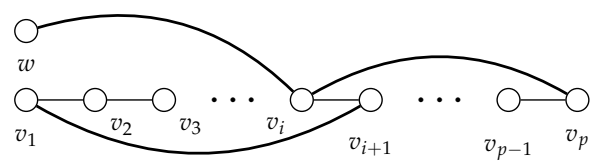


Tomando o caminho em $G$ que começa em $w$, passa por $v_{i}$ e continua ao longo do ciclo $C$, obtemos um caminho mais longo que $P$, o que é um absurdo, pois supomos que $P$ era um caminho de comprimento máximo. Logo $G$ é hamiltoniano.

Notamos facilmente que a condição de Dirac não é necessária, pois, por exemplo, um ciclo $C_{n}$ com $n$ vértices é trivialmente hamiltoniano, mas $\delta\left(C_{n}\right)=2$. Por outro lado, chamamos a atenção que o Teorema de Dirac não pode ser enfraquecido. Por exemplo, considere o grafo $G$ de ordem $n=2 q$ desconexo com duas componentes completas $K_{q}$. O grau mínimo de $G$ é $q-1=\frac{n}{2}-1$. Porém $G$ não é hamiltoniano, pois é desconexo.

Teorema 3.2 (Ore (1960)). Se G é um grafo de ordem $n \geq 3$ tal que $d(u)+d(v) \geq n$ para todo par u e v de vértices não-adjacentes, então G é hamiltoniano.

Demonstração. A demonstração é semelhante à prova do Teorema 3.1. Para garantirmos que G é conexo, vamos mostrar que quaisquer dois vértices $u$ e $v$ não-adjacentes estão em uma mesma componente. Isso de fato acontece, pois como $d(u)+d(v) \geq n$ e o conjunto $S=V-\{u, v\}$ tem $n-2$ elementos, pelo princípio da casa dos pombos pelo menos um vértice do conjunto $S$ será adjacente a $u$ e $v$. Assim temos $G$ conexo. Tomamos um caminho de comprimento máximo com extremidades $u$ e $v$. Se $u$ e $v$ forem adjacentes, temos um ciclo hamiltoniano. Se $u$ e $v$ não forem adjacentes, obtemos o absurdo na desigualdade (2) pois $n \leq d(u)+d(v) \leq n-1$.

Podemos perceber que a condição de Dirac implica a condição de Ore também para vértices adjacentes. De fato, seja $G$ um grafo de ordem $n \geq 3$ tal que $\delta(G) \geq \frac{n}{2}$. Tomamos dois vértices $u$ e $v$ em $G$ onde $d(v) \geq \frac{n}{2}$ e $d(v) \geq \frac{n}{2}$ assim $d(u)+d(v) \geq \frac{n}{2}+\frac{n}{2} \geq n$. Logo $d(u)+d(v) \geq n$. O enunciado do Teorema de Ore utiliza exatamente as hipóteses necessárias para que a prova do Teorema de Dirac funcione.

O próximo resultado é uma versão mais fraca do Teorema de Ore, que garante a existência de um caminho hamiltoniano. Este resultado será utilizado para demonstrar condições espectrais para hamiltonicidade.

Lema 3.2. Seja um grafo $G$ de ordem $n \geq 3$ tal que

$$
d(u)+d(v) \geq n-1,
$$

para todo par de vértices não adjacentes u e v. Então G contém um caminho hamiltoniano.

Demonstração. Seja G um grafo satisfazendo as condições do enunciado.

Construímos um novo grafo $H$ com $n+1$ vértices composto pelo grafo $G$ e acrescentando-se um novo vértice $x$ conectado a todos os vértices de $G$.

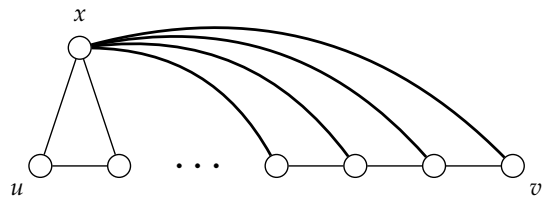

Por hipótese, $G$ satisfaz a condição de que $d_{G}(u)+d_{G}(v) \geq n-1$, para $u$ e $v$ vértices não-adjacentes em $G$, então

$$
\underbrace{d_{H}(u)}_{d_{G}(u)+1}+\underbrace{d_{H}(v)}_{d_{G}(v)+1} \geq n+1=|V(H)| .
$$

Dessa forma, temos que $d_{H}(u)+d_{H}(v) \geq|V(H)|$, para todo par de vértices não adjacentes $u$ e $v$ em $H$. Assim, pelo Teorema de Ore, $H$ é hamiltoniano. Podemos escolher um ciclo hamiltoniano $C=x v_{1} v_{2} \ldots v_{n} x$ em $H$, logo $v_{1} v_{2} \ldots v_{n}$ é um caminho hamiltoniano em $G$.

Destacamos que o Lema 3.2 não pode ser enfraquecido, pois, se substituirmos $n-1$ em (3) por $n-2$, perdemos a garantia de existir um caminho hamiltoniano no grafo. Por exemplo, se $G$ é um grafo de ordem $n=r+s$, desconexo com duas componentes completas $K_{r}$ e $K_{s}$, todo vértice $x$ de $K_{r}$ tem grau $d(x)=r-1$ e todo vértice $y$ de $K_{s}$ tem grau $d(y)=s-1$. Então $d(x)+d(y)=r-1+s-1=n-2$. Porém, $G$ não possui um caminho hamiltoniano, pois é desconexo.

Uma outra condição clássica que garante que um grafo seja hamiltoniano é a condição das arestas. 
Teorema 3.3. Seja G um grafo com m arestas. Se $m \geq\left(\begin{array}{c}n-1 \\ 2\end{array}\right)+2$, então $G$ é hamiltoniano.

Demonstração. Vamos mostrar que a condição das arestas implica a condição de Ore, que nos garante a existência de um ciclo hamiltoniano no grafo. Seja $G$ um grafo satisfazendo a condição das arestas e sejam $u$ e $v$ vértices não adjacentes de G. Assim, o número máximo de arestas em $G$ é $d(u)+d(v)+\left(\begin{array}{c}n-2 \\ 2\end{array}\right)$. Então:

$$
d(u)+d(v)+\left(\begin{array}{c}
n-2 \\
2
\end{array}\right) \geq m \geq\left(\begin{array}{c}
n-1 \\
2
\end{array}\right)+2 .
$$

Logo $d(u)+d(v) \geq\left(\begin{array}{c}n-1 \\ 2\end{array}\right)-\left(\begin{array}{c}n-2 \\ 2\end{array}\right)+2=n$.

Na Figura 12, apresentamos três grafos hamiltonianos $G, H$ e $I$ para exemplificar as relações entre a condição de Dirac, a condição de Ore e a condição das arestas.
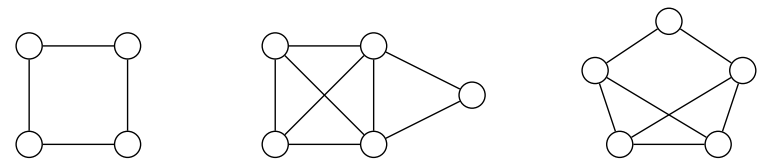

Figura 12: Grafos G, H e I, respectivamente.

Em $G$ temos um ciclo de comprimento 4 satisfazendo a condição de Dirac. Como satisfaz a condição de Dirac, $G$ também satisfaz a condição de Ore, mas a condição das arestas não é satisfeita. No grafo $H$, temos um grafo completo $K_{4}$ com a adição de um vértice de grau 2 adjacente a dois vértices de $K_{4}$. Neste exemplo podemos notar que as hipóteses do Teorema de Dirac não são satisfeitas. Já as condições das arestas e de Ore são satisfeitas. Por último, o grafo I que satisfaz a condição de Ore, mas não satisfaz a condição de Dirac nem a condição das arestas.

\subsection{Condição Necessária}

Após termos apresentado na seção anterior algumas condições suficientes clássicas para o problema de hamiltonicidade em grafos, mostraremos uma condição necessária de West (1996) para um grafo ser hamiltoniano.

Proposição 3.1. Se $G=(V, E)$ tem um ciclo hamiltoniano, então, para cada conjunto não vazio $S \subseteq V$, o grafo $G-S$ tem no máximo $|S|$ componentes.

Demonstração. Dado um ciclo hamiltoniano $C$ no grafo $G$, seja $S$ um conjunto não vazio tal que $S \subseteq V$. Para todo vértice $x \in S$ no ciclo $C, d_{C}(x)=2$. Então:

$$
2 c(G-S) \underbrace{\leq}_{(2)} E_{c}(S, G-S) \underbrace{\leq}_{(3)} 2|S|,
$$

onde $c(G-S)$ denota o número de componentes de $G-S$ e $E_{c}(S, G-S)$ é o número de arestas do ciclo com uma extremidade em $S$ e outra $G-S$. Em (2) temos que cada componente de $G-S$ é conectada a $S$ por pelo menos duas arestas do ciclo. Em (3), cada vértice de $S$ é conectado a vértices de $G-S$ por no máximo duas arestas do ciclo. Logo $c(G-S) \leq|S|$.

Assim $S$ deve ter no mínimo a mesma quantidade de vértices que as componentes de $G-S$.

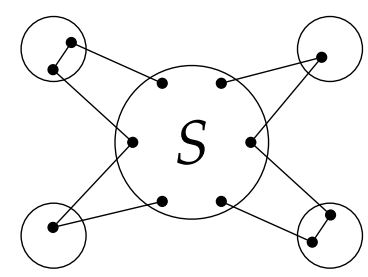

Figura 13: Componente $S$ com $|S|=6$ e $G-S$ tem 4 componentes. 
Exemplo 3.1. No exemplo da Figura 14, dado um grafo $G$, escolhemos um conjunto $S$ (composto pelos vértices pintados de preto), com cardinalidade $|S|=2$. Notamos que o grafo $G-S$ tem 3 componentes. Como a cardinalidade de $S$ é menor que o número de componentes do grafo $G-S$, o grafo $G$ não é hamiltoniano.
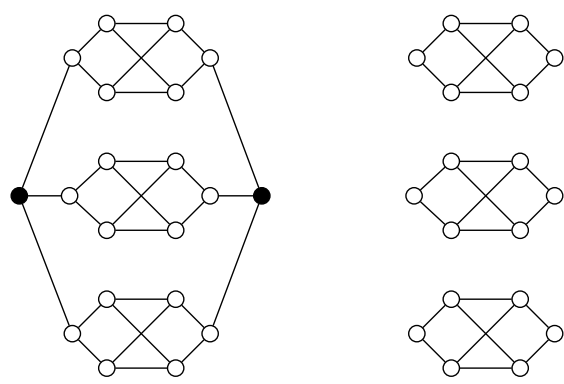

Figura 14: Grafo $G$ e grafo $G-S$.

O grafo $H$ da Figura 15 mostra que a condição necessária da Proposição 3.1 não é suficiente.

Nesse exemplo, o grafo satisfaz a condição necessária mas não possui um ciclo hamiltoniano. Todas as arestas incidentes aos vértices de grau dois deveriam ser usadas em um ciclo hamiltoniano, mas três delas são incidentes ao vértice central.

\section{Condições de Fiedler-Nikiforov}

Na seção anterior, apresentamos um apanhado de condições suficientes clássicas e uma condição necessária para que um grafo seja hamiltoniano.

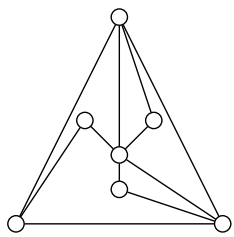

Figura 15: Grafo $H$.

Nesta seção, vamos estudar o problema de hamiltonicidade com ênfase em condições espectrais. O uso de técnicas algébricas na solução de problemas combinatórios tem uma longa história. Métodos algébricos mostraram-se particularmente eficazes no estudo de estruturas regulares e simétricas, ou na construção explícita de estruturas com uma dada propriedade, por exemplo, o estudo de grafos expansores Hoory et al. (2006).

Além disso, matrizes constituem uma estrutura de dados natural para armazenar grafos, o que motiva a busca de relações entre propriedades quantitativas e qualitativas de grafos e propriedades das matrizes que os representam. Um aspecto particularmente estudado é o espectro dessas matrizes, isto é, o estudo do conjunto de autovalores e autovetores das matrizes associadas a eles.

Nesse sentido, o trabalho de Fiedler e Nikiforov Fiedler e Nikiforov (2010) é precursor na obtenção de condições suficientes espectrais para hamiltonicidade. A partir desse trabalho, destacamos resultados espectrais obtidos recentemente para classes específicas de grafos, como grafos bipartidos Liu et al. (2015); Lu et al. (2012) e para autovalores de outras matrizes Yu e Fan (2013). 


\subsection{Os resultados de Fiedler - Nikiforov}

Fiedler e Nikiforov encontraram condições suficientes para a hamiltonicidade de um grafo com base no seu raio espectral.

Seja um grafo $G$ com $n$ vértices e $\mu(G)$ o maior autovalor da matriz de adjacências de $G$. Escrevemos $K_{n-1} \cup v$ para a união de um grafo completo de $n-1$ vértices e um vértice isolado, e $K_{n-1} \odot e$ para um grafo completo de $n-1$ vértices com uma aresta pendente. A Figura 16 mostra os dois tipos de grafos citados acima.

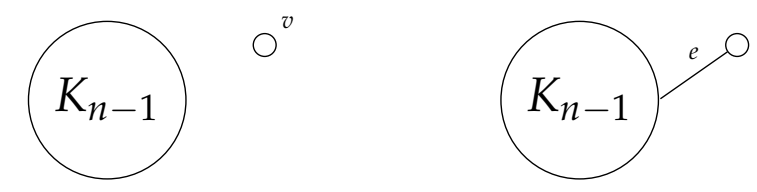

Figura 16: Grafos $K_{n-1} \cup v$ e $K_{n-1} \odot e$.

Notamos que $K_{n-1} \cup v$ não tem caminho hamiltoniano pois é desconexo e que $K_{n-1} \odot e$ não tem ciclo hamiltoniano pois tem um vértice de grau 1.

Fiedler e Nikiforov (2010) demonstraram os seguintes teoremas:

Teorema 4.1. Se $\mu(G) \geq n-2$, então $G$ contém um caminho hamiltoniano, exceto quando $G=K_{n-1} \cup v$. Se $\mu(G)>n-2$, então $G$ contém um ciclo hamiltoniano, exceto quando $G=K_{n-1} \odot e$.

Um resultado semelhante foi obtido em termos do grafo complementar de G.

Teorema 4.2. Se $\mu(\bar{G}) \leq \sqrt{n-1}$, então $G$ contém um caminho hamiltoniano exceto quando $G=K_{n-1} \cup v$. Se $\mu(\bar{G}) \leq$ $\sqrt{n-2}$, então $G$ contém um ciclo hamiltoniano exceto quando $G=K_{n-1} \odot e$.

Para demonstrar os resultados acima, os autores utilizaram condições clássicas apresentadas na seção 3, como o Teorema de Ore e a condição das arestas, por exemplo. Além disso, foram necessários definições e resultados preliminares. O conceito de fecho do grafo e suas propriedades e as desigualdades de Stanley (1987) e Hofmeister (1988) também foram importantes para as demonstrações, que apresentaremos nas próximas seções.

\subsection{Demonstração do Teorema 4.1}

Nesta seção apresentaremos resultados importantes para a demonstração do Teorema 4.1. Primeiramente mostramos um resultado semelhante ao Teorema 3.3 (condição das arestas) e depois disso demonstramos a desigualdade de Stanley (1987).

Lema 4.1. Seja $G$ um grafo com $n \geq 6$ vértices e $m$ arestas. Se $m \geq\left(\begin{array}{c}n-1 \\ 2\end{array}\right)$ então $G$ contém um caminho hamiltoniano, exceto quando $G=K_{n-1} \cup v$. Se a desigualdade for estrita, então $G$ contém um ciclo hamiltoniano, exceto quando $G=K_{n-1} \odot e$.

Demonstração. Seja $G$ um grafo satisfazendo a condição das arestas $m \geq\left(\begin{array}{c}n-1 \\ 2\end{array}\right)$. Sejam $u$ e $v$ vértices não adjacentes de $G$ e seja $H=G-\{u, v\}$. Consideramos dois casos:

(i) $H$ não é completo. Basta mostrar que a condição $d(u)+d(v) \geq n-1$ é satisfeita.

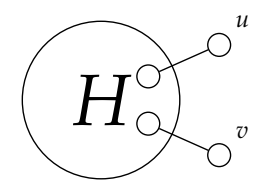

Neste caso, o número máximo de arestas de $G$ é $d(u)+d(v)+\left(\begin{array}{c}n-2 \\ 2\end{array}\right)-1$. Logo,

$$
d(u)+d(v)+\left(\begin{array}{c}
n-2 \\
2
\end{array}\right)-1 \geq m \geq\left(\begin{array}{c}
n-1 \\
2
\end{array}\right),
$$


o que implica que

$$
d(u)+d(v) \geq\left(\begin{array}{c}
n-1 \\
2
\end{array}\right)-\left(\begin{array}{c}
n-2 \\
2
\end{array}\right)+1 .
$$

Logo $d(u)+d(v) \geq n-1$. Assim, pelo Lema 3.2, G possui um caminho hamiltoniano e, se a desigualdade for estrita, pelo Teorema 3.2, G possui um ciclo hamiltoniano.

(ii) $H$ é completo

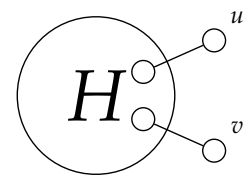

Então o número máximo de arestas de $G$ é $d(u)+d(v)+\left(\begin{array}{c}n-2 \\ 2\end{array}\right)$. Logo,

$$
\begin{gathered}
d(u)+d(v)+\left(\begin{array}{c}
n-2 \\
2
\end{array}\right) \geq m \geq\left(\begin{array}{c}
n-1 \\
2
\end{array}\right) \\
\text { o que implica que } d(u)+d(v) \geq\left(\begin{array}{c}
n-1 \\
2
\end{array}\right)-\left(\begin{array}{c}
n-2 \\
2
\end{array}\right) .
\end{gathered}
$$

Portanto,

$$
d(u)+d(v) \geq n-2
$$

Como $n \geq 6$, temos que $d(u)+d(v) \geq 4$. Ressaltamos que se $d(u)=0$ e $d(v) \geq 4$, nesse caso temos um grafo $G=K_{n-1} \cup u$, que satisfaz a condição das arestas mas não tem um caminho hamiltoniano. Se $d(u)=1 \mathrm{e}$ $d(v) \geq 3$ temos o grafo $G=K_{n-1} \odot e$, que satisfaz a condição das arestas mas não tem um ciclo hamiltoniano. Note que podemos encontrar facilmente um caminho hamiltoniano em $G$. Pois pela qualidade dos graus dos vértices $u$ e $v$ garantimos que saímos de $u$ percorremos os vértices de $K_{n-2}$ e chegamos ao vértice $v$.

Para garantirmos um ciclo hamiltoniano, temos a desigualdade estrita em (4). Portanto, $d(u)+d(v) \geq 5$. Suponhamos, sem perda de generalidade, que $d(u) \geq 2$ e $d(v) \geq 3$. Note que o grau do vértice $v$ ser no mínimo 3 garante que haja ao menos um vértice adjacente a $v$ e não-adjacente a $u$. Tomamos um ciclo que começa no vértice $u$, entra em $K_{n-2}$ pelo vértice $v_{j}$ e percorre $K_{n-2}$ até algum vértice $v_{i}$ adjacente ao vértice $v$. Após, saímos de $v$ e entramos novamente em $K_{n-2}$ pelo vértice $v_{m}$ e percorremos $K_{n-2}$ (sem repetir os vértices já utilizados) até o vértice $v_{n}$ adjacente ao vértice $u$.

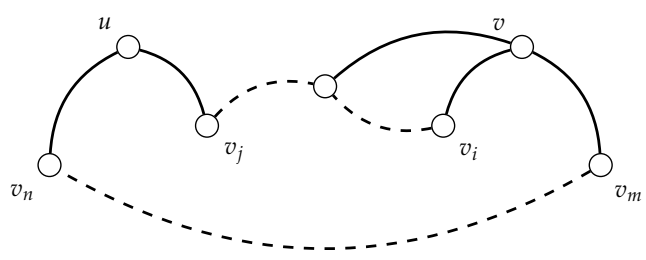

Observamos que a hipótese $n \geq 6$ no Lema 4.1 é necessária. Destacamos que o lema não é satisfeito para um caso específico para $n=4$ e um específico para $n=5$. No caso $n=4$, se tomarmos $G=K_{1,3}$ e $u$ e vértices não-adjacentes temos um contra-exemplo para o caso em que $m=3$ que não apresenta um caminho hamiltoniano.

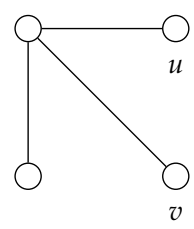

Figura 17: $G=K_{1,3}$. 
Já no caso $n=5$, se tomarmos o grafo $G$ da Figura 18, em que $m=7$, temos um contra-exemplo, pois $G$ não apresenta um ciclo hamiltoniano apesar de satisfazer a desigualdade estrita.

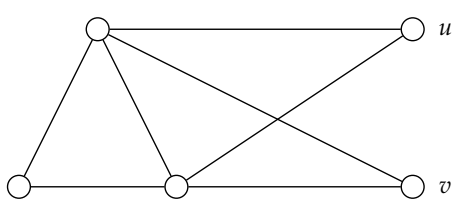

Figura 18: Contra-exemplo.

A desigualdade de Stanley (1987) que apresentamos a seguir é importante para a demonstração do Teorema 4.1 dada em Fiedler e Nikiforov (2010), pois ela apresenta uma relação entre o raio espectral $\mu(G)$ da matriz de adjacências e o número de arestas $m$ do grafo $G$.

\subsubsection{Desigualdade de Stanley}

Em seu artigo, Stanley (1987) relaciona o raio espectral da matriz de adjacências de um grafo com o número de arestas do grafo.

Teorema 4.3 (Desigualdade de Stanley). Seja G um grafo com m arestas e A a matriz de adjacências de G. Então

$$
\mu(A) \leq \frac{1}{2}(-1+\sqrt{1+8 m}),
$$

e a igualdade é válida se, e somente se, $G$ é a união disjunta de um grafo completo $K_{k}$ e vértices isolados, onde $k$ é um inteiro positivo.

Para a demonstração, Stanley utiliza um resultado de Brualdi e Hoffman (1985) que mostra que o raio espectral $\mu(A)$ satisfaz $\mu(A) \leq k-1$, valendo a igualdade se, e somente se, existe uma matriz $P$ de permutação tal que $P A P^{T}$ tem a forma

$$
\left[\begin{array}{cc}
J_{k}^{0} & 0 \\
0 & 0
\end{array}\right]
$$

onde $J_{k}^{0}$ é uma matriz de ordem $k \times k$ com 0 's na diagonal principal e 1's nas demais componentes. Em outras palavras, isso significa que $G$ é isomorfo à união disjunta de um grafo completo $K_{k}$ e vértices isolados.

Note que o Teorema 4.3 fornece um limite superior para o raio espectral de qualquer grafo com $m$ arestas, o qual implica o resultado em Brualdi e Hoffman (1985) no caso particular em que $m=\left(\begin{array}{l}k \\ 2\end{array}\right)$.

Demonstração do Teorema 4.3. Seja $A$ a matriz de adjacências do grafo $G$. Denotamos por $A_{i}$ a linha $i$ da matriz $A$ e $r_{i}$ a soma dos elementos da linha $A_{i}$. Assim:

$$
A=\left[\begin{array}{c}
A_{1} \\
A_{2} \\
\vdots \\
A_{i} \\
\vdots \\
A_{n}
\end{array}\right]_{n \times 1}
$$

Seja $x=\left(x_{1}, \ldots, x_{n}\right)^{T}$ um autovetor de $A$ com $\|x\|=1$ correspondendo ao autovalor $\mu(A)$, de forma que $A x=\mu(A) x$. Tomamos um vetor $x(i)$ obtido de $x$ pela substituição do elemento $x_{i}$ por zero, isto é, $x(i)=$ $(x_{1}, x_{2}, \ldots, \underbrace{0}_{x_{i}}, \ldots, x_{n})^{T}$. Sabendo que $A x=\mu(A) x$, temos que $A_{i} x=\mu(A) x_{i}$. Como os elementos da diagonal principal de $A$ são nulos, então temos que $A_{i} x=A_{i} x(i)$, logo $\mu(A) x_{i}=A_{i} x(i)$.

Assim, pela desigualdade de Cauchy-Schwartz, 


$$
\mu(A)^{2} x_{i}^{2}=\left|A_{i} x(i)\right|^{2} \leq \underbrace{\left\|A_{i}\right\|^{2}}_{*} \cdot \underbrace{\|x(i)\|^{2}}_{* *}=r_{i}\left(1-x_{i}^{2}\right),
$$

para $1 \leq i \leq n$. Em $(*)$, temos o vetor $A_{i}=\left(a_{i 1}, a_{i 2}, \ldots, a_{i n}\right)$ e por hipótese $r_{i}=a_{i 1}+a_{i 2}+\cdots+a_{i n}$.

Como $A_{i}$ é um vetor que representa a linha $i$ da matriz de adjacências, então é formado apenas por zeros e 1's. Ao elevarmos ao quadrado cada termo, a soma não se altera. Logo $\left\|A_{i}\right\|^{2}=a_{i 1}^{2}+a_{i 2}^{2}+\cdots+a_{i n}^{2}=r_{i}$. Em $(* *)$, temos o vetor $x=\left(x_{1}, x_{2}, \ldots, x_{i}, \ldots x_{n}\right)$. Como $\|x\|=1$, então

$$
\begin{gathered}
1=x_{1}^{2}+x_{2}^{2}+\cdots+x_{i}^{2}+\cdots+x_{n}^{2} . \text { Logo } \\
1-x_{i}^{2}=x_{1}^{2}+x_{2}^{2}+\cdots+x_{i-1}^{2}+x_{i+1}^{2}+\cdots+x_{n}^{2} .
\end{gathered}
$$

Portanto, $1-x_{i}^{2}=\|x(i)\|^{2}$. Isso justifica (6).

Somando (6) em relação a $i$, obtemos

$$
\mu(A)^{2} \sum_{i=1}^{n} x_{i}^{2} \leq \sum_{i=1}^{n} r_{i}-\sum_{i=1}^{n} r_{i} x_{i}^{2}
$$

Mas $\sum_{i=1}^{n} x_{i}^{2}=1$ e $\sum_{i=1}^{n} r_{i}=r_{1}+r_{2}+\cdots+r_{n}=2 m$, pois esta é a soma das linhas da matriz de adjacências, então

$$
\mu(A)^{2} \leq 2 m-\sum_{i=1}^{n} r_{i} x_{i}^{2}
$$

Agora analisando $\sum_{i=1}^{n} r_{i} x_{i}^{2}$, temos

$$
\begin{gathered}
\sum_{i=1}^{n} r_{i} x_{i}^{2}=\sum_{i, j}^{n} x_{i}^{2} a_{i j} \underbrace{=}_{(\star)} \sum_{i<j}^{n}\left(x_{i}^{2}+x_{j}^{2}\right) a_{i j} \underbrace{\geq}_{(\star \star)} \sum_{i<j}^{n} 2 x_{i} x_{j} a_{i j} \\
\underbrace{=}_{(\star \star \star \star)} \sum_{i, j}^{n} x_{i} a_{i j} x_{j}=x^{T} A x .
\end{gathered}
$$

Observe que $(\star)$ é válido pela simetria da matriz de adjacências, em $(\star \star$ ) a desigualdade ocorre pois $\left(x_{i}-x_{j}\right)^{2}=x_{i}^{2}-2 x_{i} x_{j}+x_{j}^{2}$. Novamente pela simetria de $A$, garantimos

Como $A x=\mu(A) x$, temos que $x^{T} A x=x^{T} \mu(A) x=\mu(A) x^{T} x=\mu(A)$ pois $x^{T} x=\|x\|=1$. Logo $\sum_{i=1}^{n} r_{i} x_{i}^{2} \geq$ $\mu(A)$, por (8). De (7) resulta

$$
\mu(A)^{2}-2 m \leq-\sum_{i=1}^{n} r_{i} x_{i}^{2} \leq-\mu(A) .
$$

Então $\mu(A)^{2}+\mu(A)-2 m \leq 0$. Usando conceitos básicos de inequação de $2^{\circ}$ grau concluímos que a desigualdade acima resulta em (5).

Para garantir a igualdade em (5), todas as desigualdades no argumento devem ser igualdades. Em particular, nós temos que

$$
\left(x_{i}^{2}+x_{j}^{2}\right) a_{i j}=2 x_{i} x_{j} a_{i j}
$$

para todo $i<j$, o que implica que $\left(x_{i}-x_{j}\right)^{2} a_{i j}=0$. Portanto, $a_{i j}=0$ ou $x_{i}=x_{j}$. Assim escolhemos uma matriz $P$ de permutação tal que $P x$ tem a forma

$$
P x=\left(y_{1}, y_{1}, \ldots, y_{1}, y_{2}, y_{2}, \ldots, y_{2}, \ldots, y_{j}, y_{j}, \ldots, y_{j}\right),
$$

onde $y_{1}, y_{2}, \ldots, y_{j}$ são distintos. Isso significa dizer que a matriz $P$ altera a rotulação, agrupando os elementos iguais de $x$. Afirmamos que $P A P^{T}$ será uma matriz em blocos na forma 


$$
P A P^{T}=\left[\begin{array}{cccc}
B_{1} & & & 0 \\
& B_{2} & & \\
& & \ddots & \\
0 & & & B_{j}
\end{array}\right] .
$$

De fato, sabemos que $\left(y_{i}-y_{j}\right)^{2} a_{i j}=0$ e que $y_{i} \neq y_{j}$ por definição. Assim $a_{i j}=0$, logo teremos apenas zeros nas entradas fora dos blocos. Cada bloco $B_{i}$ está associado a um autovetor $\left(y_{i}, y_{i}, \ldots, y_{i}\right)^{T}$, dessa forma, $(1,1, \ldots, 1)^{T}$ é um autovetor de $B_{i}$. Assim cada $B_{i}$ tem a mesma soma nas linhas, então $\mu(A)$ é o máximo na soma das linhas de $A$. Portanto $\sqrt{1+8 m}=j$ é um inteiro ímpar, e

$$
1+8 m=j^{2} \Rightarrow e=\frac{j^{2}-1}{8}=\frac{\frac{j^{2}-1}{4}}{2}=\frac{\frac{(j+1)}{2} \cdot \frac{(j-1)}{2}}{2} .
$$

Para $k=\frac{j+1}{2}$, temos $m=\left(\begin{array}{l}k \\ 2\end{array}\right)$. Assim ao substituirmos $m=\left(\begin{array}{l}k \\ 2\end{array}\right)$ em (5) obtemos $\mu(A)=k-1$ e de Brualdi e Hoffman (1985) segue que existe um bloco não nulo $B_{1}=J_{k}^{0}$, completando a demonstração.

Nesse momento, temos as ferramentas necessárias para demonstrar o Teorema 4.1.

Demonstração do Teorema 4.1. Pelo Teorema 4.3 temos

$$
\mu(G) \leq-\frac{1}{2}+\sqrt{2 m+\frac{1}{4}}
$$

e, por hipótese, temos que

$$
\mu(G) \geq n-2
$$

Assim,

$$
\begin{gathered}
n-2 \leq \mu(G) \leq-\frac{1}{2}+\sqrt{2 m+\frac{1}{4}} \\
\Rightarrow n-2 \leq-\frac{1}{2}+\sqrt{2 m+\frac{1}{4}} \\
n-2+\frac{1}{2} \leq \sqrt{2 m+\frac{1}{4}} \\
\Rightarrow\left(n-\frac{3}{2}\right)^{2}-\frac{1}{4} \leq 2 m \Rightarrow \frac{n^{2}-3 n+2}{2}=\left(\begin{array}{c}
n-1 \\
2
\end{array}\right) \leq m .
\end{gathered}
$$

Como $m \geq\left(\begin{array}{c}n-1 \\ 2\end{array}\right)$, pelo Lema 4.1 garantimos que $G$ possui um caminho hamiltoniano exceto quando $G=K_{n-1} \cup v$. Se a desigualdade em (9) for estrita, garantimos que $G$ possui um ciclo hamiltoniano exceto quando $G=K_{n-1} \odot e$. Isso completa a demonstração do Teorema 4.1.

\subsection{Demonstração do Teorema 4.2}

A demonstração do Teorema 4.2 é baseada no conceito de k-fecho de um grafo, usada diretamente por Ore (1960) e formalmente introduzida por Bondy e Chvátal (1976). Também utilizaremos a desigualdade de Hofmeister (1988). Nas próximas seções, apresentaremos esses conceitos e resultados que serão importantes para a compreensão da demonstração.

\subsection{O fecho de um grafo}

Os resultados que apresentaremos nesta seção são inspirados no Teorema de Ore (1960). Primeiramente mostraremos um resultado cuja a prova é semelhante à do Teorema 3.1.

Lema 4.2. (Bondy-Chvátal) Sejam $G$ um grafo e u e v vértices não-adjacentes de $G$ tais que $d_{G}(u)+d_{G}(v) \geq n$. Então $G$ é hamiltoniano se, e somente se, $G+$ uv é hamiltoniano. 
Demonstração. $(\Rightarrow)$ É imediata, já que, se $G$ é hamiltoniano, então $G+u v$ também é hamiltoniano.

$(\Leftarrow)$ Supõe que $G^{\prime}=G+u v$ é um grafo hamiltoniano. Então existe um ciclo em $G^{\prime}$ não repetindo nenhum vértice. Se esse ciclo não usa a aresta $u v$ então teremos um ciclo hamiltoniano em $G$. Se esse ciclo usa a aresta $u v$, então, em $G$, temos um caminho hamiltoniano com extremidades $u$ e $v$. Como, por hipótese, $d_{G}(u)+d_{G}(v) \geq n$, podemos estender esse caminho para um ciclo pelo método da demonstração do Teorema 3.1. Logo $G$ tem um ciclo hamiltoniano.

O Lema 4.2 motiva a definição do conceito de fecho de um grafo.

Definição 4.1. O fecho de um grafo $G$ com $n$ vértices, denotado $C_{n}(G)$, é o grafo obtido de $G$ de forma recursiva pela adição de arestas entre pares de vértices não adjacentes $u$ e $v$ tais que

$$
d(u)+d(v) \geq n
$$

Destacamos que a ordem na qual as arestas são adicionadas a $G$ formando o fecho não tem efeito no resultado final, e portanto o fecho de um grafo $G$ é bem definido. A Figura 19 ilustra a formação do fecho de um grafo $G$ com seis vértices. Neste exemplo acontece que o fecho é completo.

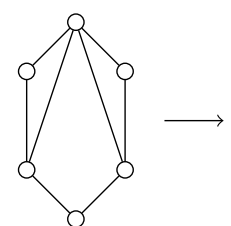

G
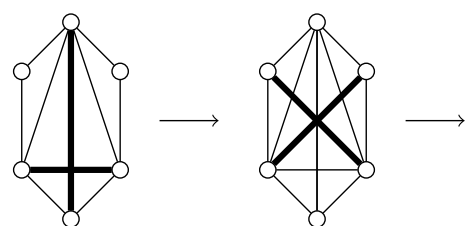

Figura 19: Fecho do grafo G.

O estudo da operação fecho para o problema de ciclos hamiltonianos gerou o seguinte resultado de Bondy e Chvátal (1976).

Teorema 4.4. Um grafo $G$ é hamiltoniano se, e somente se, o seu fecho $C_{n}(G)$ é hamiltoniano.

Corolário 4.1. Seja G um grafo de ordem $n \geq 3$ cujo fecho é completo. Então G é hamiltoniano.

Definição 4.2. O k-fecho de um grafo $G$, denotado por $C_{k}(G)$, é o grafo obtido de $G$ por um processo recursivo, o qual consiste em adicionar arestas para pares de vértices não adjacentes $u, v$ tais que

$$
d(u)+d(v) \geq k
$$

Teorema 4.5. Um grafo $G$ tem um caminho hamiltoniano se, e somente se, $C_{n-1}(G)$ tem um caminho hamiltoniano.

Se assumirmos que $H=C_{k}(G)$ não é completo, dentre todos os vértices de $H$ tal que o grau é menor do que $n-1$, escolhemos um vértice com maior grau e denotamos por $v$. Dentre todos os vértices não adjacentes a $v$, escolhemos um com maior grau e denotamos por $u$. Pela definição de $k$-fecho temos

$$
d_{H}(u)+d_{H}(v)<k, \operatorname{logo} d_{H}(u)+d_{H}(v) \leq k-1 .
$$

O fato de que vértices não-adjacentes do $k$-fecho satisfazem (10) é utilizado para a demonstração do Teorema 4.2.

\subsection{Desigualdade de Hofmeister}

Além dos conceitos do fecho de um grafo $G$ definidos acima, também utilizaremos a desigualdade de Hofmeister (1988) para a demonstração do Teorema 4.2.

Em Hofmeister (1988), foi determinado um limite inferior para o raio espectral $\mu(G)$ em relação à sequência de graus de $G$. Iá existem na literatura diversos resultados sobre a relacão entre o raio espectral $u(G)$ e a seauência 
de graus de um grafo G. Por exemplo, segue da teoria de matrizes não negativas que o raio espectral satisfaz a desigualdade

$$
\delta(G) \leq \mu(G) \leq \Delta(G)
$$

A igualdade é válida no lado esquerdo de (11) se o grafo $G$ é regular; no lado direito, se $G$ tem uma componente $\Delta$-regular.

Outro limite inferior para $\mu(G)$ é dado por Von Collatz e Sinogowitz (1957). Eles mostram que

$$
\mu(G) \geq \frac{1}{n} \sum_{i=1}^{n} d_{i}
$$

onde $d=\left(d_{1}, \ldots, d_{n}\right)$ é a sequência de graus de $G$. Para grafos regulares

$$
\mu(G)=\frac{1}{n} \sum_{i=1}^{n} d_{i}=\frac{1}{n}\left(d_{1}+d_{2}+d_{3}+\ldots+d_{n}\right)
$$

temos que

$$
\mu(G)=\frac{1}{n}(n \cdot d)=d .
$$

Para compreendermos o resultado principal de Hofmeister, temos que apresentar algumas definições preliminares.

Definição 4.3. Sejam o vetor $x=\left(x_{1}, \ldots, x_{n}\right)$ com entradas não negativas. Para $p \geq 1$, a p-média de $x$ é definida por

$$
x^{(p)}=\left(\frac{1}{n} \sum_{i=1}^{n} x_{i}^{p}\right)^{1 / p},
$$

desde que $\lim _{p \rightarrow \infty} x(p)=\max \left\{x_{1}, \ldots, x_{n}\right\}$.

Lema 4.3. Hofmeister (1986) A p-média, vista como uma função de p, é contínua e estritamente crescente se x possuir pelo menos duas componentes diferentes.

Para definirmos a média espectral característica, tomamos agora um grafo G. As desigualdades (11), (12) e o Lema 4.3 implicam a existência de um único $p \in \mathbb{R}_{\geq 1} \cup\{\infty\}$ tal que $d^{(p)}=\mu(G)$, desde que $G$ não seja regular. Esse $p$ é chamado de média espectral característica de $G$, que denotamos por $\operatorname{char}(G)$.

Apresentamos o resultado de Hofmeister que será utilizado na demonstração do Teorema 4.2.

Teorema 4.6 (Desigualdade de Hofmeister). Para todo grafo $G$, temos char $(G) \geq 2$.

Demonstração. Seja $G$ um grafo com sequência de graus $\left(d_{1}, \ldots, d_{n}\right)$. Para obter nosso resultado basta mostrar que

$$
\mu(G) \geq d^{(2)}=\left(\frac{1}{n} \sum_{i=1}^{n} d_{i}^{2}\right)^{1 / 2},
$$

pelo Lema 4.3. Sejam o vetor unitário $x_{0}=1 / \sqrt{n} \cdot(1, \ldots, 1)^{T}$ e a $A$ matriz de adjacências do grafo $G$. É fácil ver que

$$
\left\|A x_{0}\right\|^{2}=\frac{1}{n} \sum_{i=1}^{n} d_{i}^{2}
$$

Assim $d^{(2)}=\left\|A x_{0}\right\| \leq \underbrace{\sup }_{\|x\|=1}\|A x\|=\mu(G)$. Então $\mu(G) \geq d^{(2)}$, como queríamos demonstrar.

Após apresentarmos o conceito do fecho de um grafo e suas proprieades e a desigualdade de Hofmeister, temos todas as ferramentas necessárias para demonstrar o Teorema 4.2.

Demonstração do Teorema 4.2. Seja $H=C_{n-1}(G)$. Suponhamos que $\mu(\bar{G}) \leq \sqrt{n-1}$, mas que $G$ não tem um caminho hamiltoniano. Pelo Teorema 4.5, o grafo $H=C_{n-1}(G)$ não tem um caminho hamiltoniano. Utilizando a propriedade principal (10) do $k$-fecho de um grafo $G$, temos que $d_{H}(u)+d_{H}(v) \leq n-2$ para todo par de vértices não adjacentes $u$ e $v$ em $H$.

$$
\begin{gathered}
d_{\bar{H}}(u)+d_{\bar{H}}(v)=n-1-d_{H}(u)+n-1-d_{H}(v) \\
d_{\bar{H}}(u)+d_{\bar{H}}(v)-2 n+2=\underbrace{-d_{H}(u)-d_{H}(v)}_{\star} .
\end{gathered}
$$


Em $\star$ temos que $d_{H}(u)+d_{H}(v) \leq n-2$. Então

$$
\begin{gathered}
d_{\bar{H}}(u)+d_{\bar{H}}(v)-2 n+2 \geq-n+2 \\
d_{\bar{H}}(u)+d_{\bar{H}}(v) \geq n
\end{gathered}
$$

para toda aresta $u v \in E(\bar{H})$. Agora, somando essas desigualdades para todas arestas $u v \in E(\bar{H})$, obtemos

$$
\sum_{u v \in E(\bar{H})} d_{\bar{H}}(u)+d_{\bar{H}}(v) \geq n e(\bar{H})
$$

Cada termo $d_{\bar{H}}(u)$ aparece na soma $d_{\bar{H}}(u)$ vezes

$$
\sum_{v \in V(\bar{H})} d_{\bar{H}}^{2}(v)=\sum_{u v \in E(\bar{H})} d_{\bar{H}}(u)+d_{\bar{H}}(v) \geq n e(\bar{H}) .
$$

Usando a desigualdade de Hofmeister demonstrada no Teorema 4.6, obtemos

$$
\begin{gathered}
n \mu^{2}(\bar{H}) \geq \sum_{u \in V(\bar{H})} d_{\bar{H}}^{2}(u) \geq n e(\bar{H}) \\
n \mu^{2}(\bar{H}) \geq n e(\bar{H}) .
\end{gathered}
$$

Uma vez que $\bar{H} \subset \bar{G}$, nós temos, por hipótese,

$$
\mu(\bar{H}) \leq \mu(\bar{G}) \leq \sqrt{n-1} .
$$

Portanto,

$$
n \mu^{2}(\bar{H}) \leq n(n-1)
$$

De (13), temos que

$$
n(n-1) \geq n \mu^{2}(\bar{H}) \geq n e(\bar{H})
$$

e agora facilmente podemos ver que $e(\bar{H}) \leq n-1$ e

$$
e(H)=\left(\begin{array}{l}
n \\
2
\end{array}\right)-e(\bar{H}) \geq\left(\begin{array}{c}
n-1 \\
2
\end{array}\right) .
$$

Como supomos que $H$ não tem caminho hamiltoniano e temos que $e(H) \geq\left(\begin{array}{c}n-1 \\ 2\end{array}\right)$, o Lema 4.1 , implica que $H=K_{n-1} \cup v$. Se $G=H$ a prova está completa. Então assumimos que $G$ é um subgrafo de $K_{n-1} \cup v$. Então $\bar{G}$ é uma estrela $K_{1, n-1}$ de ordem $n$ com algumas arestas adicionadas e, em particular, é conexo. Assim pelo Teorema de Perron-Frobenius e pelo Corolário 2.1

$$
\mu(\bar{G})>\mu\left(K_{1, n-1}\right)=\sqrt{n-1}
$$

contradizendo $\mu(\bar{G}) \leq \sqrt{n-1}$ e completando a prova para caminhos hamiltonianos. Agora, suponha que $\mu(\bar{G}) \leq$ $\sqrt{n-2}$, mas $G$ não tem um ciclo hamiltoniano, usando o Teorema 4.4 e os argumentos acima nós vimos que

$$
e(H)>\left(\begin{array}{c}
n-1 \\
2
\end{array}\right)
$$

e uma vez que $H$ não tem um ciclo hamiltoniano, o Lema 4.1, implica que $H=K_{n-1} \odot e$. Se $G=H$, a prova está completa, então assumimos que $G$ é um subgrafo de $K_{n-1} \odot e$. Então $\bar{G}$ é uma estrela $K_{1, n-2}$ com algumas arestas adicionadas. Portanto $\bar{G}$ contém um subgrafo de $K_{1, n-2}$. Assim pelo Teorema de Perron-Frobenius e pelo Corolário 2.1

$$
\mu(\bar{G})>\mu\left(K_{1, n-2}\right)=\sqrt{n-2},
$$

contradizendo $\mu(\bar{G}) \leq \sqrt{n-2}$ e completando a demonstração. 


\section{Condição de Butler- Chung}

\subsection{Butler - Chung}

Butler e Chung (2010) mostram uma condição espectral suficiente para um grafo ser hamiltoniano com base em uma análise da proximidade entre os autovalores da matriz laplaciana e o grau médio do grafo.

Teorema 5.1. Seja G um grafo com n vértices com grau médio de e $0=\lambda_{n}^{L} \leq \lambda_{n-1}^{L} \leq \lambda_{n-2}^{L} \leq \ldots \leq \lambda_{1}^{L}$ os autovalores da matriz laplaciana de $G$. Existe uma constante $c$ tal que, para $n$ suficientemente grande, se

$$
\left|d-\lambda_{i}^{L}\right| \leq c \frac{(\log \log n)^{2}}{\log n(\log \log \log n)} d
$$

para todo $i \neq n$, então $G$ é hamiltoniano.

Quando $G$ é $d$-regular, pela Proposição 2.2 temos a seguinte relação entre os autovalores da matriz de adjacências e os autovalores da matriz laplaciana:

$$
\lambda_{i}^{A}=d-\lambda_{n-i+1}^{L} .
$$

Como o grafo é $d$-regular, temos que $\lambda_{1}^{A}=d$ está relacionado com o autovalor $\lambda_{n}^{L}$, que é o único autovalor que não é levado em consideração no enunciado do Teorema 5.1. E com isso vemos que o Teorema 5.1 implica o seguinte resultado de Krivelevich e Sudakov (2003), que foi obtido para a constante $c=\frac{1}{1000}$.

Teorema 5.2. Seja $G=(V, E)$ um grafo d-regular com n vértices, onde $n$ é suficientemente grande. Se

$$
\left|\lambda_{i}^{A}\right| \leq \frac{(\log \log n)^{2}}{1000 \log n(\log \log \log n)} d
$$

para todo $i \neq 1$, então $G$ é hamiltoniano.

O resultado do Teorema 5.1 é mais forte do que o Teorema 5.2, pois garante a hamiltonicidade para qualquer grafo, enquanto que Teorema 5.2 garante hamiltonicidade apenas para grafos $d$-regulares.

Quando $G$ não é regular, os autovalores das matrizes de adjacências e laplaciana não estão mais relacionadas de uma forma trivial e torna-se mais importante determinar qual espectro é utilizado. Na prova do Teorema 5.1, é utilizado o espectro da matriz laplaciana, pois a matriz laplaciana é útil para controlar a expansão da vizinhança de um conjunto de vértices.

Destacamos que a demonstração que vamos apresentar do Teorema 5.1 adapta proposições e lemas para grafos $d$-regulares que são utilizados na demonstração do Teorema 5.2. Por simplicidade, muitos dos lemas técnicos são demonstrados para o caso $d$-regular.

\subsection{A família de $(n, d, \lambda)$-grafos}

Um grafo $G$ é chamado $(n, d, \lambda)$-grafo se, e somente se é $d$-regular, tem $n$ vértices e $\lambda_{2}^{A}=\lambda$. Essa é uma família de grafos pseudo-aleatórios, veja Krivelevich e Sudakov (2006) para mais informações. Nesta seção apresentamos uma quantidade de resultados na distribuição das arestas em grafos pseudo-aleatórios. Para um subconjunto de vértices $U \subset V(G)$ denotamos por $N(U)$ o conjunto de todos os vértices em $V-U$ adjacentes a algum vértice em $U$. Também denotamos por $e(U)$ o número de arestas com ambas as extremidades em $U$.

Teorema 5.3. (Alon e Spencer, 2004, Corolário 9.2.5, 9.2.6) Seja $G=(V, E)$ um $(n, d, \lambda)$-grafo. Então:

(a) para quaisquer dois subconjuntos $B, C \subset V$ o número de arestas de $G$ com uma extremidade em $B$ e outra em $C$ satisfaz

$$
\left|e(B, C)-\frac{|B||C| d \mid}{n}\right| \leq \lambda \sqrt{|B||C|\left(1-\frac{|B|}{n}\right)} ;
$$


(b) para todo subconjunto $B \subseteq V$,

$$
\left|e(B)-\frac{|B|^{2} d}{2 n}\right|<\frac{\lambda|B|}{2} \text {. }
$$

Podemos destacar um trabalho mais recente de Krivelevich e Sudakov (2006) que apresenta uma cota melhor do que (17).

A seguir mostraremos proposições que serão importantes para a demonstração do Teorema 5.1. As Proposições 5.1, 5.2 e 5.3 são ditas propriedades da expansão das arestas. A Proposição 5.1 diz que, para um conjunto de vértices $V_{0}$ pequeno, o número de arestas com ambas as extremidades em $V_{0}$ é limitado por $\lambda\left|V_{0}\right|$. Isso nos garante que temos arestas com uma extremidade em $V_{0}$ e outra extremidade em $V-V_{0}$. De fato, como $\sum_{v \in V_{0}} d(v)=\left|e\left(V_{0}, V-V_{0}\right)\right|+2\left|e\left(V_{0}\right)\right|$, esse número é dado por $e\left(V_{0}, V-V_{0}\right) \geq d\left|V_{0}\right|-2 \lambda\left|V_{0}\right|$.

A Proposição 5.1 a seguir garante que muitas arestas saem de qualquer conjunto pequeno. Porém, como diversas arestas podem ter a mesma extremidade, isso não garante que o conjunto de vizinhos seja grande. A Proposição 5.2 garante que para todo conjunto de vértices muito pequeno $V_{0}$, teremos uma vizinhança relativamente grande.

Nas proposições abaixo, supomos que $G=(V, E)$ é um $(n, d, \lambda)$-grafo $\operatorname{com} \lambda<\frac{d}{2}$.

Proposição 5.1. Todo subconjunto $V_{0} \subset V$ de cardinalidade $\left|V_{0}\right| \leq \frac{\lambda n}{d}$ gera no máximo $\lambda\left|V_{0}\right|$ arestas.

Demonstração. De (18),

$$
e\left(V_{0}\right) \leq \frac{\left|V_{0}\right|^{2} d}{2 n}+\frac{\lambda\left|V_{0}\right|}{2}
$$

como $\left|V_{0}\right| \leq \frac{\lambda n}{d}$, temos

$$
e\left(V_{0}\right) \leq \frac{\lambda n}{d} \frac{\left|V_{0}\right| d}{2 n}+\frac{\lambda\left|V_{0}\right|}{2}=\lambda\left|V_{0}\right|
$$

$\operatorname{Logo} e\left(V_{0}\right) \leq \lambda\left|V_{0}\right|$

Proposição 5.2. Para todo subconjunto $V_{0} \subset V$ de cardinalidade $\left|V_{0}\right| \leq \frac{\lambda^{2} n}{d^{2}}$

$$
\left|N\left(V_{0}\right)\right|>\frac{(d-2 \lambda)^{2}}{3 \lambda^{2}}\left|V_{0}\right| \text {. }
$$

Demonstração. Denotamos $N\left(V_{0}\right)=U$. Pela Proposição 5.1 aplicada a $V_{0}$, temos $e\left(V_{0}\right) \leq \lambda\left|V_{0}\right|$. Como o grau de todo vértice em $V_{0}$ é $d$, nós obtemos:

$$
e\left(V_{0}, U\right) \geq d\left|V_{0}\right|-2 e\left(V_{0}\right) \underbrace{\geq}_{\text {Proposição } 5.1} d\left|V_{0}\right|-2 \lambda\left|V_{0}\right| .
$$

Por outro lado, segue de (17) que

Da desigualdade (19), temos

$$
e\left(V_{0}, U\right)<\frac{\left|V_{0}\right||U| d}{n}+\lambda \sqrt{\left|V_{0}\right||U|}
$$

$$
e\left(V_{0}, U\right) \geq(d-2 \lambda)\left|V_{0}\right|
$$

e isso implica que

$$
\frac{\left|V_{0}\right||U| d}{n}+\lambda \sqrt{\left|V_{0}\right||U|}>(d-2 \lambda)\left|V_{0}\right|
$$

Se $|U| \leq \frac{(d-2 \lambda)^{2}}{3 \lambda^{2}}\left|V_{0}\right|$, então

$$
\frac{\left|V_{0}\right||U| d}{n}+\lambda \sqrt{\left|V_{0}\right||U|}
$$

$$
\leq \frac{\left|V_{0}\right|(d-2 \lambda)^{2}\left|V_{0}\right| d}{3 \lambda^{2} n}+\lambda \sqrt{\frac{\left|V_{0}\right|(d-2 \lambda)^{2}\left|V_{0}\right|}{3 \lambda^{2}}}
$$




$$
\begin{gathered}
=\frac{\left|V_{0}\right|^{2}(d-2 \lambda)^{2} d}{3 \lambda^{2} n}+\lambda \frac{\left|V_{0}\right|(d-2 \lambda)}{\sqrt{3} \lambda} \\
=\frac{\left|V_{0}\right|^{2}(d-2 \lambda)^{2} d}{3 \lambda^{2} n}+\frac{\left|V_{0}\right|(d-2 \lambda)}{\sqrt{3}} \\
\leq \frac{\lambda^{2} n}{d^{2}} \frac{(d-2 \lambda)^{2} d\left|V_{0}\right|}{3 \lambda^{2} n}+\frac{(d-2 \lambda)\left|V_{0}\right|}{\sqrt{3}} \\
=\frac{(d-2 \lambda)^{2}\left|V_{0}\right|}{3 d}+\frac{(d-2 \lambda)\left|V_{0}\right|}{\sqrt{3}} \\
<\frac{(d-2 \lambda)\left|V_{0}\right|}{3}+\frac{(d-2 \lambda)\left|V_{0}\right|}{\sqrt{3}} \\
<(d-2 \lambda)\left|V_{0}\right| .
\end{gathered}
$$

Então $\frac{\left|V_{0}\right||U| d}{n}+\lambda \sqrt{\left|V_{0}\right||U|}<(d-2 \lambda)\left|V_{0}\right|$ contradizendo (20).

Proposição 5.3. Para todo subconjunto $V_{0} \subseteq V$ de cardinalidade $\left|V_{0}\right|>\frac{\lambda^{2} n}{d^{2}},\left|N\left(V_{0}\right)\right|>\frac{n}{2}-\left|V_{0}\right|$.

Demonstração. Seja o conjunto $U=V \backslash\left(V_{0} \cup N\left(V_{0}\right)\right)$. Então claramente $e\left(V_{0}, U\right)=0$. Por outro lado de (17) temos

$$
0=e\left(V_{0}, U\right) \geq \frac{\left|V_{0}\right||U| d}{n}-\lambda \sqrt{\left|V_{0}\right||U|\left(1-\frac{|U|}{n}\right)} .
$$

Isso implica que

$$
\begin{aligned}
& \frac{\left|V_{0}\right|^{2}|U|^{2} d^{2}}{n^{2}} \leq \lambda^{2}\left(\left|V_{0}\right||U|\left(1-\frac{|U|}{n}\right)\right) \\
\Longrightarrow & \frac{\left|V_{0}\right|^{2}|U|^{2}}{\left|V_{0}\right||U|\left(1-\frac{|U|}{n}\right)} \leq \frac{\lambda^{2} n^{2}}{d^{2}} \\
\Longrightarrow & \frac{|U|}{\left(1-\frac{|U|}{n}\right)} \leq \frac{\lambda^{2} n^{2}}{d^{2}\left|V_{0}\right|}<\frac{\lambda^{2} n^{2}}{d^{2} \frac{\lambda^{2} n}{d^{2}}}=n .
\end{aligned}
$$

A desigualdade $\frac{|U|}{\left(1-\frac{|U|}{n}\right)}<n$ implica que $|U|<\frac{n}{2}$. Então $\left|N\left(V_{0}\right)\right|=|V|-\left|V_{0}\right|-|U|>\frac{n}{2}-\left|V_{0}\right|$.

Proposição 5.4. Sejam dois subconjuntos disjuntos tais que $U_{1}, U_{2} \subset V(G)$ não são conectados por uma aresta em $G$, temos $\left|U_{1}\right|\left|U_{2}\right|<\frac{\lambda^{2} n^{2}}{d^{2}}$.

Demonstração. Por (17)

$$
0=e\left(U_{1}, U_{2}\right)>\frac{\left|U_{1}\right|\left|U_{2}\right| d}{n}-\lambda \sqrt{\left|U_{1}\right|\left|U_{2}\right|}
$$

Assim $\frac{\left|U_{1}\right|\left|U_{2}\right| d}{n}<\lambda \sqrt{\left|U_{1}\right|\left|U_{2}\right|}$. Segue que $\left|U_{1}\right|\left|U_{2}\right|<\frac{\lambda^{2} n^{2}}{d^{2}}$.

Proposição 5.5. Se $G=(V, E)$ é um $(n, d, \lambda)$-grafo com $\lambda<\frac{d}{2}$ então $G$ é conexo. 
Demonstração. Se $G$ é desconexo, então $G$ tem uma componente conexa $V_{0}$ de tamanho $\left|V_{0}\right| \leq \frac{n}{2}$. Como $N\left(V_{0}\right)=\varnothing$, segue da Proposição 5.3 que $\left|V_{0}\right| \leq \frac{\lambda^{2} n}{d^{2}}$. Isso contradiz a Proposição 5.2, já que a mesma afirma que $\left|N\left(V_{0}\right)\right| \geq$ $\frac{(d-2 \lambda)^{2}\left|V_{0}\right|}{3 \lambda^{2}}>0$

Para provar o resultado do Teorema 5.1, precisaremos de um resultado mais geral, que adapta o resultado do Teorema 5.3 para a matriz laplaciana. Inicialmente, enunciaremos um resultado que é semelhante ao resultado do Teorema 5.3.

Teorema 5.4. (Butler e Chung, 2010, Teorema 2.1) Supomos que um grafo G com $n$ vértices tem grau médio d e os autovalores $0=\lambda_{n}^{L} \leq \lambda_{n-1}^{L} \leq \ldots \leq \lambda_{1}^{L}$ da matriz laplaciana satisfazem $\left|d-\lambda_{i}\right| \leq \theta$ para todo $i \neq n$. Então, para quaisquer dois subconjuntos de vértices $B$ e $C$, temos

$$
\left|e(B, C)-\frac{|B||C| d \mid}{n}\right| \leq \frac{\theta}{n} \sqrt{|B|(n-|B|)|C|(n-|C|)}
$$

$$
\left|e(B)-\frac{d|B|(|B|-1)}{2 n}\right| \leq \frac{\theta}{n}|B|(n-|B| / 2)
$$

Vamos utilizar o Teorema 5.4 para provar o resultado de Butler e Chung em dois passos. Primeiramente, mostraremos que nós temos uma boa expansão na vizinhança e depois disso mostraremos que o grafo é conexo. Para estudar essa expansão das arestas e mostrar que o grafo é conexo vamos enunciar uma proposição que apresenta resultados parecidos como das proposições acima. A demonstração dessa proposição sera omitida, pois são semelhantes as proposições já provadas anteriormente.

Proposição 5.6. (Butler e Chung, 2010, Proposição 2.3) Seja G um grafo de $n$ vértices com grau médio $d$ e $\left|d-\lambda_{i}\right| \leq \theta$ para os autovalores da matriz laplaciana tal que $i \neq n$. Assumimos que $8 \theta<d$ e que $B, C \subseteq V$. Então temos que:

(a) se $|B| \leq \frac{\theta n}{d}$, então $e(B) \leq \frac{3}{2}|B|$;

(b) se $|B| \leq \frac{\theta^{2} n}{d^{2}}$, então $|N(B)|>\frac{(d / 2-4 \theta)^{2}}{3 \theta^{2}}|B|$;

(c) se $|B|>\frac{\theta^{2} n}{d^{2}}$, então $|N(B)|>\frac{n}{2}-|B|$;

(d) se $B \cap C=\varnothing$ e e $(B, C)=0$, então $|B||C|<\frac{\theta^{2} n^{2}}{d^{2}}$;

(e) Gé conexo.

\subsection{Demonstração do Teorema 5.1}

A demonstração do Teorema 5.1 é parecida com a demonstração do Teorema 5.2, com algumas pequenas mudanças nas constantes. Destacamos que os resultados utlizados para demonstrar o resultado de Butler e Chung estão relacionados com o teorema de Krivelevich e Sudakov para grafos $d$-regulares.

A demonstração envolve uma técnica de rotação de caminhos inspirada na demonstração clássica do Teorema 3.1. A ideia principal é que, dado um caminho de comprimento máximo, as arestas dos pontos finais do caminho devem estar conectadas ao centro do caminho e podem ser usadas para rotacionar o caminho e criar um novo caminho de comprimento máximo. Esse processo de rotação pode ser repetido e nós podemos formar uma grande coleção de caminhos máximos, o que permite que um desses caminhos possa ser fechado para formar um ciclo hamiltoniano.

Demonstração Teorema 5.1. Seja $P=\left(v_{1}, v_{2}, \ldots, v_{m}\right)$ um caminho de comprimento máximo em $G$. Se $v_{i}$ é adjacente a $v_{m}$ para algum $i$ então outro caminho máximo é dado por $P^{\prime}=\left(v_{1}, \ldots, v_{i}, v_{m}, v_{m-1}, \ldots, v_{i+1}\right)$, que inclui a aresta $\left\{v_{i}, v_{m}\right\}$ e retira a aresta $\left\{v_{i}, v_{i+1}\right\}$. 


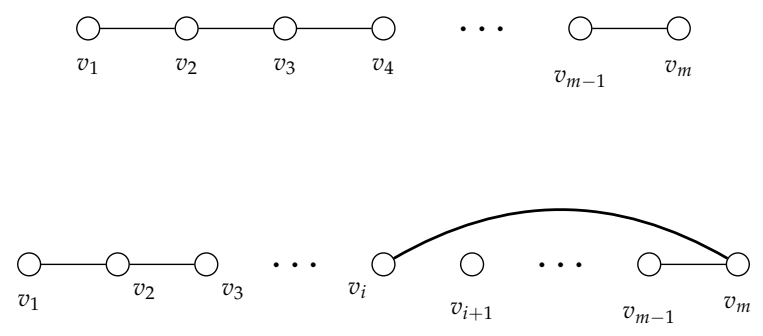

Nós dizemos que $P^{\prime}$ é uma rotação de $P$ com extremidade fixa $v_{1}$, eixo de rotação $v_{i}$ e aresta removida $\left\{v_{i}, v_{i+1}\right\}$. Em seguida, escolhemos um vértice de $P^{\prime}$ como eixo de rotação e criamos um novo caminho máximo $P^{\prime \prime}$, e assim por diante. Para $t \geq 0$ seja o conjunto $S_{t}$ de vértices $v \in\left\{v_{2}, \ldots, v_{m}\right\}$ tais que $v$ é extremidade de um caminho obtido de $P$ por no máximo $t$ rotações com extremidade fixa $v_{1}$ e todas as arestas removidas pertencentes a $P$. Então, temos que $S_{1}$ é o conjunto de vértices finais obtido através do caminho inicial usando até uma rotação, $S_{2}$ permite rotações nos caminhos de $S_{1}$, mantendo $v_{1}$ fixo e utilizando arestas de $P$, e assim sucessivamente. Logo $S_{1} \subseteq S_{2} \subseteq S_{3} \subseteq \ldots$. É fácil ver que, pela maximalidade do caminho $P$, todas as arestas incidentes a um vértice em $S_{t}$ têm a segunda extremidade no caminho $P$.

Analisando os conjuntos $S_{t}$, podemos mostrar esses têm uma boa expansão na vizinhança.

Lema 5.1. Para todo $t \geq 0,\left|S_{t+1}\right| \geq \frac{1}{2}\left|N\left(S_{t}\right)\right|-\frac{3}{2}\left|S_{t}\right|$.

Demonstração. Seja

$$
T=\left\{i \geq 2: v_{i} \in N\left(S_{t}\right), v_{i-1}, v_{i}, v_{i+1} \notin S_{t}\right\} .
$$

Vemos que, para um vértice $v_{i} \in T$, as arestas $\left\{v_{i-1}, v_{i}\right\}$ e $\left\{v_{i}, v_{i+1}\right\}$ não são arestas removidas. Assim $|T| \geq$ $\left|N\left(S_{t}\right)\right|-3\left|S_{t}\right|$, pois, para cada $v_{i} \in S_{t}$, temos que $i, i-1, i+1 \notin T$.

Consideramos um vértice $v_{i} \in V(P)$ com $i \in T$. Então, $v_{i}$ tem um vizinho $x \in S_{t}$. Isso significa que existe um caminho $P^{\prime}$ de comprimento máximo com $x$ como extremidade, obtido de $P$ por no máximo $t$ rotações. Observamos que, durante esse processo repetido de rotações, vamos removendo arestas no nosso caminho inicial $P$. Como $v_{i-1}, v_{i}, v_{i+1} \notin S_{t}$ ambas as arestas $\left\{v_{i-1}, v_{i}\right\}$ e $\left\{v_{i}, v_{i+1}\right\}$ continuam presentes em $P^{\prime}$. Agora, rotacionando $P^{\prime}$ com eixo de rotação $v_{i}$, uma das arestas $\left\{v_{i-1}, v_{i}\right\},\left\{v_{i}, v_{i+1}\right\}$ será removida. Logo $v_{i-1}$ ou $v_{i+1} \in S_{t+1}$ e, assim, cada elemento de $T$ produz um elemento de $S_{t+1}$, por exemplo, se $v_{i+1} \in S_{t+1}, v_{i+1}$ poderia ser imagem de $v_{i}$ ou $v_{i+2}$.

Temos que dois elementos de $T$ podem gerar um mesmo elemento de $S_{t+1}$, de forma que $\left|S_{t+1}\right| \geq \frac{|T|}{2}$. Então

$$
\left|S_{t+1}\right| \geq \frac{1}{2}\left|N\left(S_{t}\right)\right|-\frac{3}{2}\left|S_{t}\right|
$$

Agora seja

$$
t_{0}=\left\lceil\frac{\log n-2 \log (d / \theta)}{2 \log (d / \theta)-10}\right\rceil+2
$$

Pela Proposição 5.6(b), se $\left|S_{t}\right| \leq \frac{\theta^{2} n}{d^{2}}$ então $\left|N\left(S_{t}\right)\right| \geq \frac{(d / 2-4 \theta)^{2}\left|S_{t}\right|}{3 \theta^{2}}$. Pelo Lema 5.1 temos que

$$
\left|S_{t+1}\right| \geq \frac{(d / 2-4 \theta)^{2}\left|S_{t}\right|}{6 \theta^{2}}-\frac{3}{2}\left|S_{t}\right|
$$

Em particular, se $d$ é grande comparado a $\theta$ (digamos $d>1000 \theta$ ) então, quando $\left|S_{t}\right| \leq \frac{\theta^{2} n}{d^{2}}$, podemos ver facilmente por indução que

$$
\frac{\left|S_{t+1}\right|}{\left|S_{t}\right|} \leq \frac{(d / 2-4 \theta)^{2}}{7 \theta^{2}}
$$


Isso implica que, em no máximo

$$
\frac{\log \frac{\theta^{2} n}{d^{2}}}{\log \frac{(d / 2-4 \theta)^{2}}{7 \theta^{2}}} \leq t_{0}-2
$$

passos nós temos que $\left|S_{t}\right|>\frac{\theta^{2} n}{d^{2}}$. Pela Proposição 5.6(c) quando adicionamos um passo nós temos que

$$
\begin{aligned}
\left|S_{t+1}\right| & \geq \frac{1}{2}\left|N\left(S_{t}\right)\right|-\frac{3}{2}\left|S_{t}\right| \geq \frac{1}{2}\left(\frac{n}{2}-\left|S_{t}\right|\right)-\frac{3}{2}\left|S_{t}\right| \\
& =\frac{n}{4}-2\left|S_{t}\right| \geq \frac{n}{4}-2\left|S_{t+1}\right|
\end{aligned}
$$

Isso implica que $\left|S_{t+1}\right| \geq \frac{n}{12}$. Aplicando a Proposição $5.6 \operatorname{com} B=S_{t+1}$ e $C=V-N\left(S_{t+1}\right)$, concluímos que

$$
\begin{gathered}
\frac{n}{12}|C| \leq|B||C|<\frac{\theta^{2} n^{2}}{d^{2}} \\
\Longrightarrow|C|<\frac{12 \theta^{2} n}{d^{2}} \\
\Longrightarrow\left|N\left(S_{t+1}\right)\right| \geq n \cdot\left(1-12 \theta^{2} / d^{2}\right) .
\end{gathered}
$$

Utilizando novamente a Proposição 5.6(c) como em (26) temos

$$
\begin{aligned}
\left|S_{t+2}\right| & \geq \frac{1}{2}\left|N\left(S_{t+1}\right)\right|-\frac{3}{2}\left|S_{t+1}\right| \\
& \geq \frac{1}{2} n \cdot\left(1-12 \theta^{2} / d^{2}\right)-\frac{3}{2}\left|S_{t+2}\right|,
\end{aligned}
$$

e portanto $\left|S_{t+2}\right| \geq \frac{n \cdot\left(1-12 \theta^{2} / d^{2}\right)}{5}>\frac{n}{6}$ para $n$ suficientemente grande.

Sejam $B\left(v_{1}\right)=S_{t_{0}}$ e $A_{0}=B\left(v_{1}\right) \cup\left\{v_{1}\right\}$. Para $v \in B\left(v_{1}\right)$, podemos repetir o argumento acima e obtermos um conjunto $B(v)$ de extremidades dos caminhos de comprimento máximo com ponto fixo $v$. Assim $|B(v)|>n / 6$. Note que cada extremidade em $B(v)$ será obtida em no máximo $2 t_{0}$ rotações de $P$.

Também notamos que cada vértice em $B\left(v_{1}\right)$ está em $P$, e portanto $P$ deve conter pelo menos $n / 6$ vértices, isto é, $P$ contém uma fração constante dos vértices do grafo. Para cada $a \in A_{0}, b \in B(a)$, existe um caminho de comprimento máximo $P(a, b)$ com extremidades $a$ e $b$ que é obtido de $P$ em no máximo $\rho=2 t_{0}$ rotações.
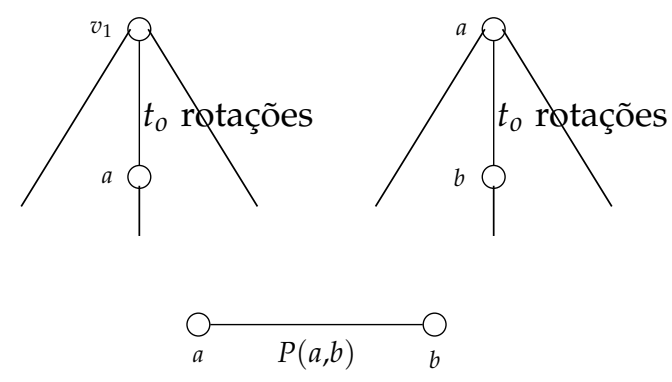

Agora retornamos ao caminho inicial $P$, que dividimos em $2 \rho$ segmentos disjuntos $I_{1}, \ldots, I_{2 \rho}$, cada um com comprimento pelo menos $\lfloor n / 12 \rho\rfloor$.

$$
P
$$<smiles>COOOOO</smiles> 
Já que cada caminho $P(a, b)$ é obtido de $P$ por no máximo $\rho$ rotações, existem no mínimo $\rho$ segmentos que não foram alterados. Chamaremos esses segmentos de intactos. Definimos a orientação natural do segmento como a orientação no caminho original $P$, quando percorrido de $v_{1}$ a $v_{n}$. Dependendo da rotação, a orientação de um segmento intacto pode mudar. Dado um segmento intacto, dizemos que o segmento tem orientação relativa + com relação a um novo caminho se mantém a orientação natural e - caso contrário. Vejamos um primeiro exemplo:

$P$
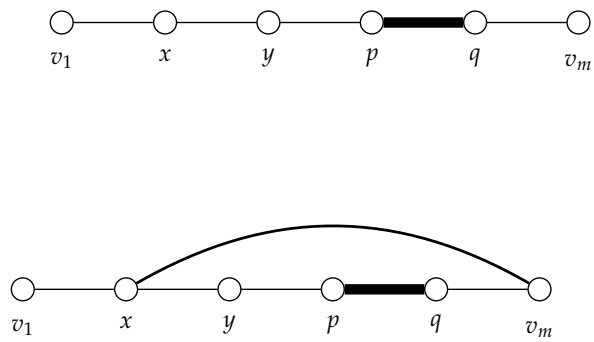

Rotacionamos o caminho inicial $P$, com eixo de rotação $x$ e a aresta removida $\{x, y\}$ criamos um novo caminho de comprimento máximo,

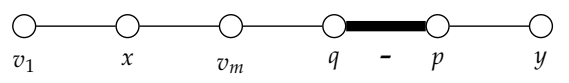

em que o segmento $p q$ trocou sua orientação relativa.

Vejamos um segundo exemplo:

P
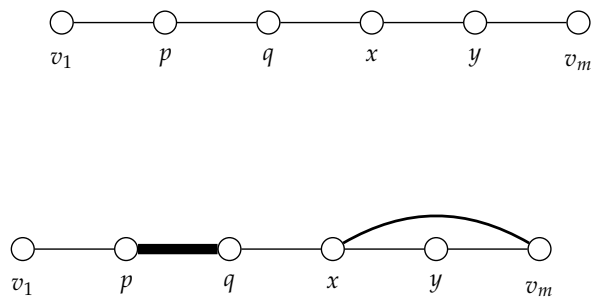

Rotacionamos o caminho inicial $P$, com eixo de rotação $x$ e a aresta removida $\{x, y\}$ criamos um novo caminho de comprimento máximo, que

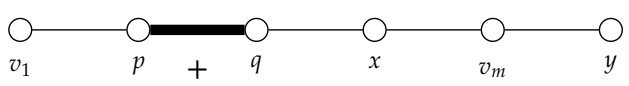

manteve a orientação relativa de $p q$.

Seja $k=2 \max \left\{1,\lceil 500 \theta \rho / d\rceil\right.$. Consideramos as sequências $\sigma=I_{i_{1}}, \ldots, I_{i_{k}}$ de $k$ segmentos intactos de $P$ que ocorrem nessa ordem em $P(a, b)$, com as respectivas orientações relativas. Dizemos então que $P(a, b)$ contém $\sigma$. Note que $P(a, b)$ tem no mínimo $\rho$ segmentos $I_{i_{j}}$ intactos, $\operatorname{logo} P(a, b)$ contém no mínimo $\left(\begin{array}{l}\rho \\ k\end{array}\right)$ sequências $\sigma$. Considere o seguinte exemplo para $k=4$.

Acima, construímos uma sequência $\sigma$ com quatro segmentos intactos de $P$ que ocorrem nessa ordem em $P(a, b)$. Note que os segmentos $I_{13}$ e $I_{12}$ mantiveram sua orientação relativa no novo caminho, o que não aconteceu com os segmentos $I_{1}$ e $I_{20}$.

Dado $\sigma$, definimos $L(\sigma)$ como o conjunto de todos os pares $(a, b)$ para os quais $\sigma$ está contido em $P(a, b)$. O número total de possibilidades para a sequência direcionada $\sigma$ é no máximo

$$
2 \rho(2 \rho-1) \cdots(2 \rho-k+1) \cdot 2^{k}
$$




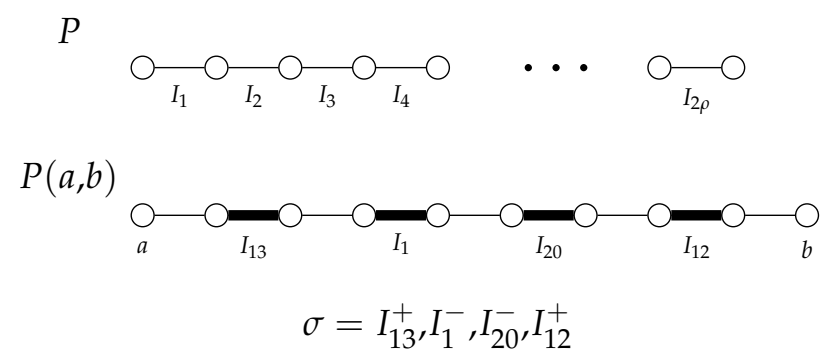

Considere a sequência $\sigma_{0}$ que está contida em $P(a, b)$ para o maior número de pares $(a, b), a \in A_{0}$ e $b \in B(a)$. Afirmamos que

$$
\begin{aligned}
\left|L\left(\sigma_{0}\right)\right| \geq & \frac{n}{6} \cdot \frac{n}{6} \cdot \frac{\left(\begin{array}{c}
\rho \\
k
\end{array}\right)}{2 \rho(2 \rho-1) \ldots(2 \rho-k+1)} \\
& >\frac{n^{2}}{36}\left(\frac{p-k}{2 \rho-k}\right)^{k} \cdot \frac{1}{k ! 2^{k}} .
\end{aligned}
$$

Existem pelo menos $\frac{n}{6} \cdot \frac{n}{6}$ maneiras de escolher o par $(a, b)$ e cada $P(a, b)$ contém no mínimo $\left(\begin{array}{l}\rho \\ k\end{array}\right)$ sequências direcionadas $\sigma$. Finalmente, existem no máximo $2 \rho(2 \rho-1) \cdots(2 \rho-k+1)$ possibilidades para $\sigma$.

Por hipótese $k \leq \rho / 2$ então $(\rho-k) /(2 \rho-k) \geq 1 / 3$ e segue que existe a sequência $\sigma_{0}$ no qual

$$
\left|L\left(\sigma_{0}\right)\right| \geq \frac{n^{2}}{36} \cdot\left(\frac{1}{3}\right)^{k} \cdot \frac{1}{k ! 2^{k}}=\frac{n^{2}}{k ! 6^{k+2}} .
$$

Seja $\alpha=\frac{1}{k ! 6^{k+2}}$. Definimos $\hat{A}$ como o conjunto de todos $a \in A_{0}$ tais que $L\left(\sigma_{0}\right)$ contém, no mínimo, $\frac{\alpha n}{2}$ pares com a como primeiro elemento.

Tomamos $a \in A_{0}$. Se $a \in \hat{A}, a$ aparece, no máximo, $n$ vezes. Se $a \notin \hat{A}, a$ aparece, no máximo, $\frac{\alpha n}{2}$ vezes. Como $\left|L\left(\sigma_{0}\right)\right| \geq \alpha n^{2}$, temos:

$$
\begin{gathered}
\alpha n^{2} \leq n \cdot|\hat{A}|+\frac{\alpha n}{2} \cdot\left(\left|A_{0}\right|-|\hat{A}|\right) \leq n \cdot|\hat{A}|+\frac{\alpha n}{2} \cdot(n-|\hat{A}|) \\
\leq n \cdot|\hat{A}|+\frac{\alpha n^{2}}{2}-\frac{\alpha n}{2} \cdot|\hat{A}| .
\end{gathered}
$$

Logo, teremos que:

$$
\alpha n^{2}-\frac{\alpha n^{2}}{2} \leq|\hat{A}| \cdot n \cdot\left(1-\frac{\alpha}{2}\right)
$$

Portanto, $\frac{\alpha n}{2\left(1-\frac{\alpha}{2}\right)} \leq|\hat{A}|$.

Como $\left(1-\frac{\alpha}{2}\right)<1$, concluímos que $\frac{\alpha n}{2} \leq|\hat{A}|$.

Para $a \in \hat{A}$, seja $\hat{B}(a)=\left\{b \in B(a),(a, b) \in L\left(\sigma_{0}\right)\right\}$. A definição de $\hat{A}$ garante que $|\hat{B}(a)| \geq \frac{\alpha n}{2}$.

Seja $C_{1}$ a união dos primeiros $\frac{k}{2}$ segmentos de $\sigma_{0}$, fixando a ordem e a orientação relativa na qual eles ocorrem ao longo de qualquer um dos caminhos $P(a, b),(a, b) \in L\left(\sigma_{0}\right)$. Seja $C_{2}$ a união dos últimos $\frac{k}{2}$ segmentos de $\sigma_{0}$.

Note que

$$
\left|C_{i}\right| \geq \frac{k}{2} \cdot\left\lfloor\frac{n}{12 \rho}\right\rfloor \geq \frac{500 \theta \rho}{d}\left\lfloor\frac{n}{12 \rho}\right\rfloor>\frac{40 \theta n}{d}
$$




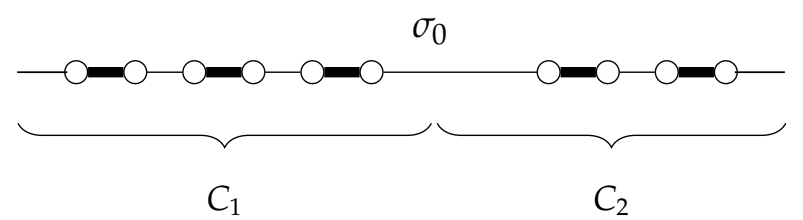

Dado um caminho $P_{0}$ e o conjunto $S \subseteq\left\{v_{1}, \ldots, v_{m}\right\}$, um vértice $v \in S$ é chamado de ponto interior de $S$ com respeito a $P$ se ambos os vizinhos de $v$ ao longo de $P$ estão em $S$. O conjunto de todos os pontos interiores de $S$ é denotado por $\operatorname{int}(S)$. Usando (24) e (27), temos o seguinte resultado:

Lema 5.2. (Krivelevich e Sudakov, 2003, Proposição 3.2) O conjunto $C_{1}$ contém um subconjunto $C_{1}^{\prime} \operatorname{com} \mid$ int $\left(C_{1}^{\prime}\right) \mid \geq \frac{n k}{48 \rho}$ tal que todo $v \in C_{1}^{\prime}$ tem, no minimo, $14 \theta$ vizinhos em int $\left(C_{1}^{\prime}\right)$. O mesmo vale para $C_{2}$.

Tal lema garante que, para um conjunto grande $C_{1}^{\prime}$, cada elemento nesse conjunto possui um grande número de vizinhos em $\operatorname{int}\left(C_{1}^{\prime}\right)$.

Agora fixamos os conjuntos $C_{1}^{\prime}$ e $C_{2}^{\prime}$. Usando a Proposição 5.6(d) é possível mostrar que os vértices das extremidades estarão ligados ao interior dos caminhos.

Lema 5.3. (Krivelevich e Sudakov, 2003, Proposição 3.3 e 3.4) Existe um vértice â $\in$ Â conectado por uma aresta ao conjunto int $\left(C_{1}^{\prime}\right)$. Similarmente, existe um vértice $\hat{b} \in \hat{B}(\hat{a})$ conectado por uma aresta ao conjunto int $\left(C_{2}^{\prime}\right)$.

Seja $x$ um vértice separando $C_{1}^{\prime}$ e $C_{2}^{\prime}$ ao longo de $P(\hat{a}, \hat{b})$. Isso divide o caminho em dois caminhos $P_{1}$ e $P_{2}$.

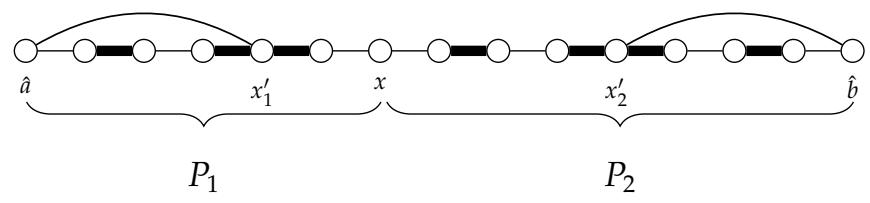

Agora fixamos $x$ e rotacionamos cada caminho $P_{i}$, garantindo que usamos somente vértices no respectivo conjunto $\operatorname{int}\left(C_{i}^{\prime}\right)$ como eixo de rotação.

Podemos mostrar que os conjuntos de extremidades são suficientemente grandes, então a Proposição 5.6(d) implica que existe uma aresta entre esses conjuntos de extremidades que chamamos de $V_{1}$ e $V_{2}$. Essa aresta fecha um caminho de comprimento máximo para um ciclo. Como $G$ é conexo, pelo Corolário 5.6(e) qualquer ciclo não-hamiltoniano pode ser estendido para um caminho adicionando algum vértice. Portanto, por hipótese, a maximalidade de $P_{0}$ implica que $P_{0}$ é um caminho hamiltoniano e, assim, criamos um ciclo hamiltoniano, como queríamos.

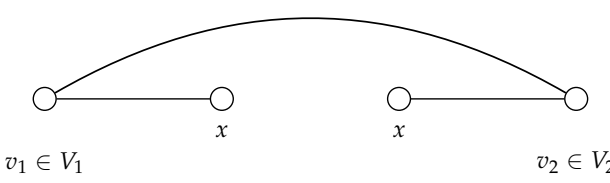

Para isso, seja o conjunto $T_{i}$ de vértices $v \in C_{1}^{\prime} \backslash x$ tais que $v$ é extremidade de um caminho obtido de $P_{1}$ por $i$ rotações com extremidade fixa $x$. Todos os eixos de rotação estão em $\operatorname{int}\left(C_{1}^{\prime}\right)$ e todas as arestas removidas estão em $P_{1}$.

Analisando os conjuntos $T_{i}$, podemos mostrar, usando (24), que, quando $\left|T_{i}\right|$ é pequeno, ele contém um subconjunto que expande, em particular, em até $\log \left(\frac{\theta n}{d}\right)$ passos, teremos $\left|T_{i}\right| \geq \frac{\theta n}{d}$.

Lema 5.4. (Krivelevich e Sudakov, 2003, Proposição 3.5) Existe um i tal que $\left|T_{i}\right| \geq \frac{\theta n}{d}$. 
Assim, o conjunto $V_{1}$ de extremidades de todas as rotações de $P_{1}$ é tal que $\left|V_{1}\right| \geq \frac{\theta n}{d}$. Pelo Lema $5.4, \hat{b}$ tem um vizinho em $\operatorname{int}\left(C_{2}^{\prime}\right)$. O mesmo argumento pode ser feito para $P_{2}$ e mostrar que o conjunto $V_{2}$ de extremidades de todas rotações de $P_{2}$ é tal que $\left|V_{2}\right| \geq \frac{\theta n}{d}$. Logo temos o seguinte:

$$
\left|V_{1}\right|\left|V_{2}\right| \geq \frac{\theta^{2} n^{2}}{d^{2}}
$$

Concluímos, pelo Corolário 5.6(d), que existe uma aresta conectando $V_{1}$ e $V_{2}$, fechando o ciclo.

\section{Conclusões}

Neste trabalho apresentamos, essencialmente, condições necessárias e suficientes para um grafo ser hamiltoniano. Tal estudo foi motivado pelo fato de tratar-se de um problema de difícil solução, apesar de ter um enunciado simples. Foram apresentadas condições suficientes clássicas, como o Teorema de Dirac - que garante hamiltonicidade via grau mínimo do grafo - e, também, o Teorema de Ore - que envolve o grau dos vértices não adjacentes. Vimos que essas condições não são necessárias, por exemplo, um ciclo $C_{n} \operatorname{com} n$ vértices é hamiltoniano, mas $\delta\left(C_{n}\right)=2$. Por outro lado, podemos mostrar que existem grafos com $n$ vértices e grau mínimo $n / 2-1$ que não são sequer conexos, quanto mais hamiltonianos, e portanto a condição de Dirac não pode ser melhorada.

A Teoria Espectral de Grafos estuda propriedades de um grafo através dos autovalores e autovetores de uma matriz associada ao grafo, que formam o espectro do grafo com respeito a essa matriz. Estudamos dois trabalhos precursores na obtenção de condições suficientes espectrais para um grafo ser hamiltoniano. Em Fiedler e Nikiforov (2010), foi obtido um resultado envolvendo a matriz de adjacências, mais especificamente, o raio espectral da matriz de adjacências. Já em Butler e Chung (2010), há uma condição envolvendo os autovalores não triviais da matriz laplaciana.

O objetivo principal deste trabalho era mostrar as condições conhecidas na literatura, bem como suas demonstrações, detalhadamente. Para apresentar as demonstrações dos resultados mais relevantes, utilizamos diversas ferramentas combinatórias e de Álgebra Linear, incluindo os conceitos de fecho de um grafo e de distribuição das arestas em grafos pseudo-aleatórios.

Percebemos também que as cotas existentes nas condições eram justas. Isso significa dizer que existem grafos para os quais as cotas não podem ser melhoradas, já estão otimizadas. Além disso, podemos destacar que existem diversos trabalhos que tratam do enfraquecimento de condições do tipo Dirac-Ore. Como sabemos, essas condições são justas, logo alguns grafos não-hamiltonianos vão ser falsos positivos em um teste mais fraco. Nesse sentido, ressaltamos o trabalho de Erdös e Hobbs (1978), que apresentam o seguinte resultado: se $n \geq 4$ e $G$ é um grafo 2 -conexo com $2 n$ ou $2 n-1$ vértices, o qual é regular de grau $n-2$, então $G$ é hamiltoniano se, e somente se, $G$ não é o grafo de Petersen.

Nos resultados espectrais estudados neste trabalho, podemos destacar uma diferença fundamental nas suas demonstrações e nos métodos utilizados. Em Fiedler e Nikiforov (2010) a cota espectral garante que um grafo seja hamiltoniano utiliza diretamente o resultado clássico de Ore e portanto qualquer grafo que satisfaça a condição espectral também satisfaz a condição de Ore. Já em Butler e Chung (2010), os autores apresentam a construção do ciclo hamiltoniano com base em propriedades do grafo que decorrem da caracterização espectral, sem a utilização de uma condição que garante hamiltonicidade. Por isso, é natutal que a demonstração do resultado de Butler e Chung seja mais difícil que a do resultado de Fiedler e Nikiforov.

\section{Referências}

Abreu, N., Del-Vecchio, R., Trevisan, V., Vinagre, C. (2014). Teoria Espectral de Grafos - Uma Introdução. SBM.

Alon, N., Spencer, J. H. (2004). The probabilistic method. John Wiley \& Sons.

Appel, K., Haken, W. (1976). Every planar map is four colorable. Bulletin of the American mathematical Society, 82(5), 711-712.

Bondy, J. A., Chvátal, V. (1976). A method in graph theory. Discrete Mathematics, 15(2), 111-135. 
Bondy, J. A., Murty, U. S. R. (2008). Graph theory, Graduate Texts in Mathematics, vol 244. Springer, New York, URL http://dx.doi.org/10.1007/978-1-84628-970-5.

Brouwer, A. E., Haemers, W. H. (2012). Spectra of graphs. Springer Science \& Business Media.

Brualdi, R. A., Hoffman, A. J. (1985). On the spectral radius of (0, 1)-matrices. Linear Algebra and its Applications, 65, 133-146.

Butler, S., Chung, F. (2010). Small spectral gap in the combinatorial Laplacian implies Hamiltonian. Ann Comb, 13(4), 403-412, URL http://dx.doi.org/10.1007/s00026-009-0039-4.

Cvetković, D. M. (1971). Graphs and their spectra. Publikacije Elektrotehničkog fakulteta Serija Matematika i fizika, $(354 / 356), 1-50$.

Cvetković, D. M., Rowlinson, P., Simić, S. (2010). An introduction to the theory of graph spectra. Cambridge University Press Cambridge.

Dirac, G. A. (1952). Some theorems on abstract graphs. Proceedings of the London Mathematical Society, 3(1), 69-81.

Erdös, P., Hobbs, A. M. (1978). A class of hamiltonian regular graphs. Journal of Graph Theory, 2(2), $129-135$.

Euler, L. (1741). Solutio problematis ad geometriam situs pertinentis. Commentarii academiae scientiarum Petropolitanae, 8, 128-140.

Fiedler, M., Nikiforov, V. (2010). Spectral radius and Hamiltonicity of graphs. Linear Algebra Appl, 432(9), 2170-2173, URL http://dx.doi.org/10.1016/j.laa.2009.01.005.

Hofmeister, M. (1986). @: Uber zusammenh@: ange zwischen valenzsequenz, eckenzahl und gr@: ossten eigenwerten von graphen und digraphen. .

Hofmeister, M. (1988). Spectral radius and degree sequence. Mathematische Nachrichten, 139(1), 37-44.

Hoory, S., Linial, N., Wigderson, A. (2006). Expander graphs and their applications. Bull Amer Math Soc (NS), 43(4), 439-561 (electronic), URL http://dx .doi .org/10.1090/S0273-0979-06-01126-8.

Hückel, E. (1931). Quantentheoretische beiträge zum benzolproblem. Zeitschrift für Physik A Hadrons and Nuclei, 70(3), 204-286.

Karp, R. M. (1972). Reducibility among combinatorial problems. Em: Complexity of computer computations (Proc. Sympos., IBMThomas J. Watson Res. Center, Yorktown Heights, N.Y., 1972), Plenum, New York, pp. 85-103.

Krivelevich, M., Sudakov, B. (2003). Sparse pseudo-random graphs are hamiltonian. Journal of Graph Theory, 42(1), 17-33.

Krivelevich, M., Sudakov, B. (2006). Pseudo-random graphs. Em: More sets, graphs and numbers, Springer, pp. $199-262$.

Liu, R., Shiu, W. C., Xue, J. (2015). Sufficient spectral conditions on hamiltonian and traceable graphs. Linear Algebra and its Applications, 467, 254-266.

Lu, M., Huiqing, L., Feng, T. (2012). Spectral radius and hamiltonian graphs. Linear Algebra and Its Applications, 437(7), 1670-1674.

Meyer, C. (2000). Matrix analysis and applied linear algebra. Society for Industrial and Applied Mathematics (SIAM), Philadelphia, PA, URL http://dx.doi.org/10.1137/1.9780898719512, with 1 CD-ROM (Windows, Macintosh and UNIX) and a solutions manual (iv+171 pp.).

Ore, O. (1960). Note on hamilton circuits. The American Mathematical Monthly, 67(1), 55-55.

Stanley, R. P. (1987). A bound on the spectral radius of graphs with e edges. Linear Algebra Appl, 87, 267-269, URL http://dx.doi.org/10.1016/0024-3795(87)90172-8.

Von Collatz, L., Sinogowitz, U. (1957). Spektren endlicher grafen. Em: Abhandlungen aus dem Mathematischen Seminar der Universität Hamburg, Springer, vol 21, pp. 63-77. 
West, D. B. (1996). Introduction to graph theory. Prentice Hall, Inc., Upper Saddle River, NJ.

Yu, G. D., Fan, Y. Z. (2013). Spectral conditions for a graph to be hamilton-connected. Em: Applied Mechanics and Materials, Trans Tech Publ, vol 336, pp. 2329-2334. 